\title{
Review Article \\ Preparation of Nanofibers with Renewable Polymers and Their Application in Wound Dressing
}

\author{
Ying Zhao, Yihui Qiu, Huanhuan Wang, Yu Chen, Shaohua Jin, and Shuseng Chen \\ School of Material Science and Engineering, Beijing Institute of Technology, Beijing 100081, China \\ Correspondence should be addressed to Yu Chen; cylsy@163.com
}

Received 11 December 2015; Accepted 17 February 2016

Academic Editor: Matheus Poletto

Copyright ( 2016 Ying Zhao et al. This is an open access article distributed under the Creative Commons Attribution License, which permits unrestricted use, distribution, and reproduction in any medium, provided the original work is properly cited.

\begin{abstract}
Renewable polymers have attracted considerable attentions in the last two decades, predominantly due to their environmentally friendly properties, renewability, good biocompatibility, biodegradability, bioactivity, and modifiability. The nanofibers prepared from the renewable polymers can combine the excellent properties of the renewable polymer and nanofiber, such as high specific surface area, high porosity, excellent performances in cell adhesion, migration, proliferation, differentiation, and the analogous physical properties of extracellular matrix. They have been widely used in the fields of wound dressing to promote the wound healing, hemostasis, skin regeneration, and treatment of diabetic ulcers. In the present review, the different methods to prepare the nanofibers from the renewable polymers were introduced. Then the recent progress on preparation and properties of the nanofibers from different renewable polymers or their composites were reviewed; the application of them in the fields of wound dressing was emphasized.
\end{abstract}

\section{Introduction}

Renewable polymers, including the polymers directly extracted from biomass and proteins, for example, starch, chitosan, cellulose, alginate, gelatin, and collagen [1], have attracted considerable attentions in the last two decades, predominantly due to their environmentally friendly properties, renewability, good biocompatibility, biodegradability, bioactivity, and modifiability [2]. The nanofibers prepared from the renewable polymers can combine the excellent properties of the renewable polymer and nanofiber [3], such as high specific surface area, high porosity, excellent performances in cell adhesion, migration, proliferation, differentiation, and the analogous physical properties of extracellular matrix (ECM). They have been widely used in the fields of wound healing, hemostasis, skin regeneration, and treatment of diabetic ulcers. In the present review, we will focus on the preparation, composition, and properties of nanofibers prepared with renewable polymers and their applications in the wound dressing. The research progress and development directions were also discussed.

\section{Methods for the Preparation of Nanofibers with Renewable Polymers}

As nanofibers are becoming increasingly important, the high throughput preparation of high quality nanofibers has drawn remarkable attentions. Currently, electrospinning is the most widely used method for the preparation of nanofibers. Other methods including Bubbfil spinning, centrifugal spinning, and freeze-drying have also been reported for their potential applications in the biological field.

2.1. Electrospinning. Electrospinning is the most commonly used method for the fabrication of nanofibers due to its simplicity, adaptability, cost-efficiency, and versatility [4].

In a typical electrospinning process, a high voltage is applied to a liquid polymer, leading to the ejection of a continuous jet strand from the spinneret towards a grounded collector. The surface tension of the polymer droplet is overcome by the applied electric field. The droplet then elongates to form a cone known as "Taylor cone" and then is extruded from the 
cone to form a fiber jet. The solvent in the jets is evaporated as the fiber jets travel through the atmosphere and solid polymer fibers are deposited on the metal collector as nonwoven web [4].

Based on the preparation process of the polymer, the electrospinning process can be classified into two groups including solution electrospinning and melt electrospinning. Solution electrospinning is limited mainly by its low productivity, extra solvent extraction process, and requirement of toxic solvent. Yet it is much more thoroughly reviewed than melt electrospinning by researchers due to more concerns on melt electrospinning, such as difficulties inherent in the finer fiber formation, higher viscosity of polymer melts, and electrical discharge issues associated with the application of high voltage [5]. Thus, several setups including multijets from single needle, multijets from multineedle, and needleless systems have been proposed for solution electrospinning to increase its productivity.

2.1.1. Multijets from Single Needle Electrospinning. For single needle electrospinning (SNE) process, a single jet is initiated from the Taylor cone formed by the application of electric field. Li et al. prepared poly(lactic acid) (PLA)/chitosan coreshell structure nanofibers from a homogeneous solution using SNE and found that this method was simpler and more effective in producing core-shell structures from homogeneous solutions than other methods [6]. Ayaz et al. successfully prepared composite nanofibers with SNE and coaxial (double needle) electrospinning and compared their performances. The polymer matrix and filler were mixed before being infused into the single needle for SNE. Therefore, the filler was uniformly distributed in the whole nanofiber. In contrast, polymer matrix and filler were infused separately into two nozzles axially centered to each other for coaxial electrospinning, which restricted the filler in the center of nanofiber. In addition, the average diameter of the nanofibers prepared by SNE is smaller than that of the nanofibers prepared by coaxial electrospinning. However, nanofibers prepared through SNE showed lower breaking elongation than those prepared by the coaxial method [7].

2.1.2. Multijets from Multineedle Electrospinning. As discussed above, SNE provides very low throughput productivity [8]. Multineedle electrospinning (MNE) system is a simple way to improve the productivity and a simple technique to fabricate composite fibers from the polymers that cannot be easily dissolved in common solvents. However, the needle configuration, number of needles, and needle gauge need to be optimized for the MNE design. For example, needles can be arranged in one- or two-dimensional configuration. However, charged jets could interfere with each other and restrain jets injected from the spinneret, leading to low production efficiency $[9,10]$. The interference between the charged jets can be resolved by several methods.

Nuryantini et al. prepared PVA/chitosan nanofiber membranes using an MNE system with a drum collector. Compared with conventional electrospinning setups, MNE setup can significantly improve the throughput while maintaining the uniformity of the size and thickness of the stacked

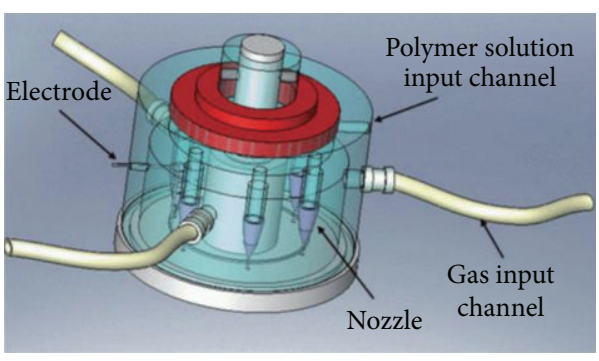

FIGURE 1: Setup of MNE spinneret assisted with sheath gas [9].

nanofibers [11]. Yu et al. fabricated nanofibers using a novel MNE setup with assistant sheath gas as shown in Figure 1 [9]. The assistant sheath gas introduced to the spinneret improved the stretching process of the charged jet and realized multijet injection. During the electrospinning, assistant gas was pumped into the chamber through gas input channels, which were uniformly distributed and thus contributed to each spinneret evenly. Sheath gas at a pressure of $50 \mathrm{kPa}$ decreased the average diameter of the nanofibers from $600 \mathrm{~nm}$ to $472.8 \mathrm{~nm}$ and decreased the diameter distribution region from $249 \mathrm{~nm}$ to $188 \mathrm{~nm}$. The assistant sheath gas also promoted the stability of the electrospinning and the uniformity of the nanofibers due to its ability to reduce the required voltage for jet injection and jet surface charge density [9].

2.1.3. Multijets from Needleless Electrospinning. MNE system usually needs a big space and optimization of the relative distance between needles to avert strong charge repulsions between the jets. Therefore, needleless electrospinning has been developed and widely used due to its notable ability to increase the production of nanofibers. For the needleless electrospinning, an electric field is applied on a large area, producing numerous polymeric jets from free liquid surface $[5,8]$.

Sasithorn and Martinová prepared silk nanofibers by both needle electrospinning and needleless electrospinning and found that the mass production rate of needleless electrospinning was much higher than that of needle electrospinning. In addition, the production rate of needleless electrospinning could be increased significantly with the increase of solution concentration and applied voltage. However, nanofibers produced by needle electrospinning were much smaller and had a narrow diameter distribution [12].

Wang et al. prepared uniform nanofibers using a needleless electrospinning system with a rotating spiral wire coil as the spinneret (Figure 2). The polymer solution was charged with a high voltage and loaded onto the coil surface by slowly rotating the coil at $40 \mathrm{rpm}$. They found the production of nanofiber was improved by increasing the length $(D)$ and diameter $(\phi)$ of the coil or decreasing the spiral distance $(d)$ and the diameter $\left(\phi_{w}\right)$ of the wire. However, these factors showed no significant effect on the average diameter of the nanofibers. Compared with the needle electrospinning, the needless electrospinning with coil spinneret showed better performance, such as producing thinner nanofibers and higher productivity [13]. 


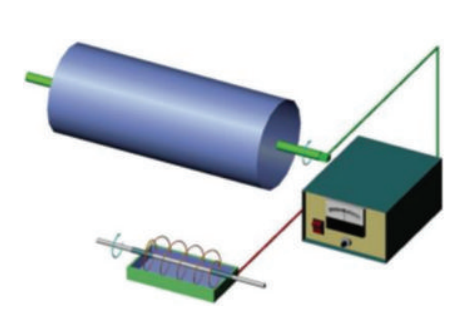

(a)

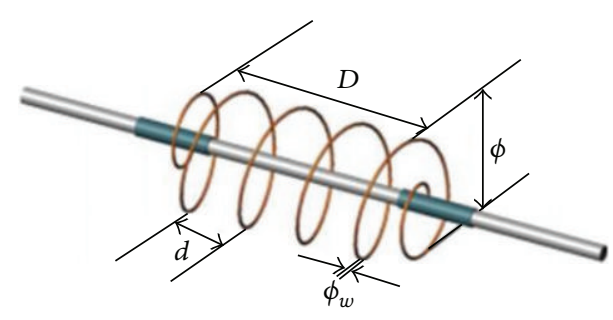

(b)

FIgURE 2: (a) Schematics of spiral coil electrospinning setup; (b) magnified view of the coil [13].

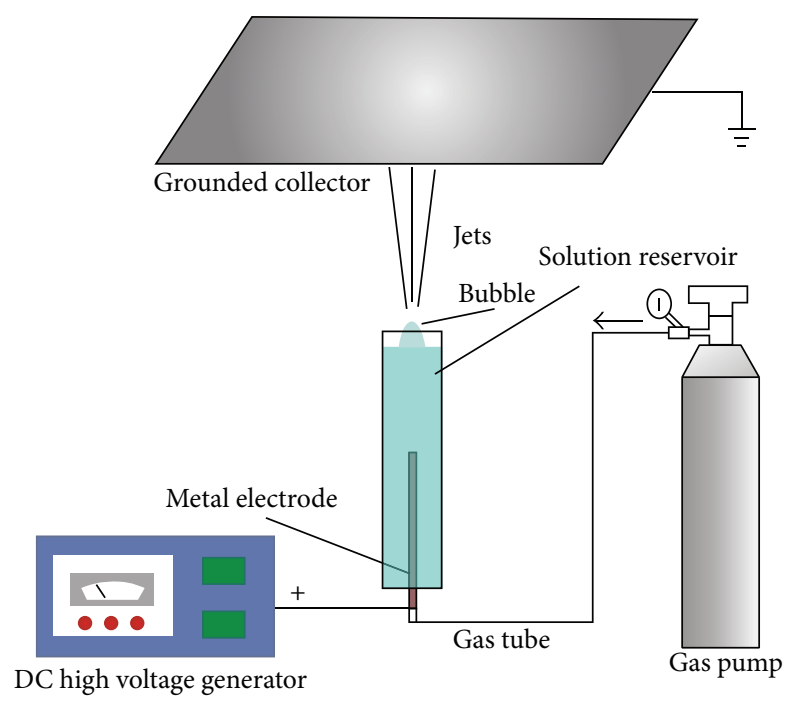

Figure 3: Bubble electrospinning setup for tunable bubble size [14].

2.2. Bubbfil Spinning. It is known that the increases in the molecular weight and viscosity of polymers lead to higher surface tension of their Taylor cone, which cannot be used to fabricate nanofibers by the conventional electrospinning methods. Therefore, higher electrostatic force is required for the electrospinning to fabricate desired nanofibers [14]. Other drawbacks of electrospinning including low productivity and uncontrollability of the spinning process call for the development of other technologies [15].

Bubbfil spinning including bubble electrospinning and blown bubble spinning is one of main newly developed methods for the preparation of nanofibers.

2.2.1. Bubble Electrospinning. Bubble electrospinning has attracted considerable attention due to its high throughput. Unlike conventional electrospinning, bubble electrospinning uses electrostatic force rather than Taylor cone to overcome the surface tension of a polymer bubble $[16,17]$. The electrospinnability of bubble electrospinning depends geometrically on the sizes of the obtained bubbles, which avoid using high voltages and thus make it the best candidate in the green production. Figure 3 shows a typical bubble electrospinning setup.

In a typical bubble electrospinning process, bubbles are formed on the surface of a polymer solution by a controllable syringe pump as the gas pump slowly turned on. The number of the bubbles is reduced with the decrease of the tube diameter. Multiple jets are then ejected from the bubbles to the grounded collector when the applied voltage surpasses a critical value. The fluid jets are usually solidified into fibers in less than 0.001 seconds $[5,14]$.

Jiang et al. mass-produced nanofibers with an improved microbubble electrospinning system where a pyramidshaped copper spinneret was used as the generator. They found that the increase of bubble size from $29.6 \pm 8.2 \mu \mathrm{m}$ to $152.7 \pm 22.7 \mu \mathrm{m}$ led to an increase in the average diameter of nanofibers from $139 \pm 39$ to $165 \pm 26 \mathrm{~nm}$. The microbubble electrospinning produced nanofibers with average diameter of $147 \pm 28 \mathrm{~nm}$ with the bubble sizes of $59.9 \pm 10.8 \mu \mathrm{m}$, which are similar to those produced by the conventional electrospinning with the same processing parameters. The throughput of microbubble electrospinning reached $8.9 \mathrm{~g} / \mathrm{h}$, which was much higher than that of the conventional electrospinning $(5.2 \mathrm{~g} / \mathrm{h})[18]$.

2.2.2. Blown Bubble Spinning. Blown bubble spinning produces nanofibers using blowing air and has been used commercially as a one-step process to convert polymer resin directly into a nonwoven mat of fibers. Figure 4 shows an experimental setup of blown bubble spinning.

Blown bubble spinning process is very much similar to bubble electrospinning except that it uses blowing air instead of electronic force to produce fine fibers [19]. However, a weak electronic field can significantly improve the performance of the blown bubble spinning, which actually turns into an electrostatic-field-assisted blown bubble spinning (Figure 5). It can also be called as air-jet assisted bubble electrospinning, depending on which one is the major functional force [14].

Li et al. prepared silk fibroin through blown bubble spinning and found that the direction of blowing air, both perpendicular (setup I in Figure 6(a)) and parallel (setup II in Figure 6(b)) to the ejected direction of broken polymer bubble, could affect the morphology, structure, and arrangement of the produced fibers. For example, random oriented nanofibers with an average diameter of $524.25 \pm 142.41 \mathrm{~nm}$ were formed through setup I and well organized fiber bundles were obtained with setup II [16].

2.3. Centrifugal Spinning. Centrifugal spinning, also called rotary spinning or rotational jet spinning, has been well developed. Since no high voltage is required by the centrifugal 


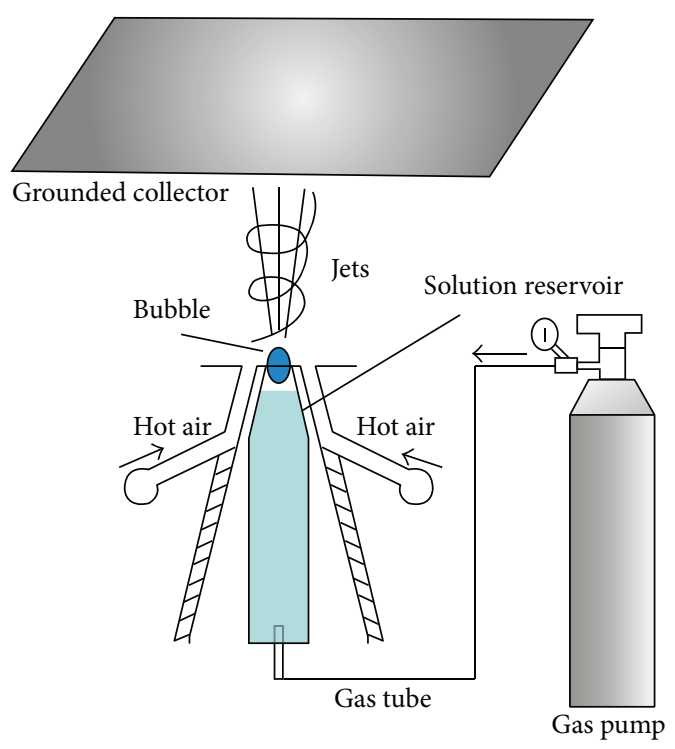

FIGURE 4: Blown bubble spinning (the only acting force is blowing air) [14].

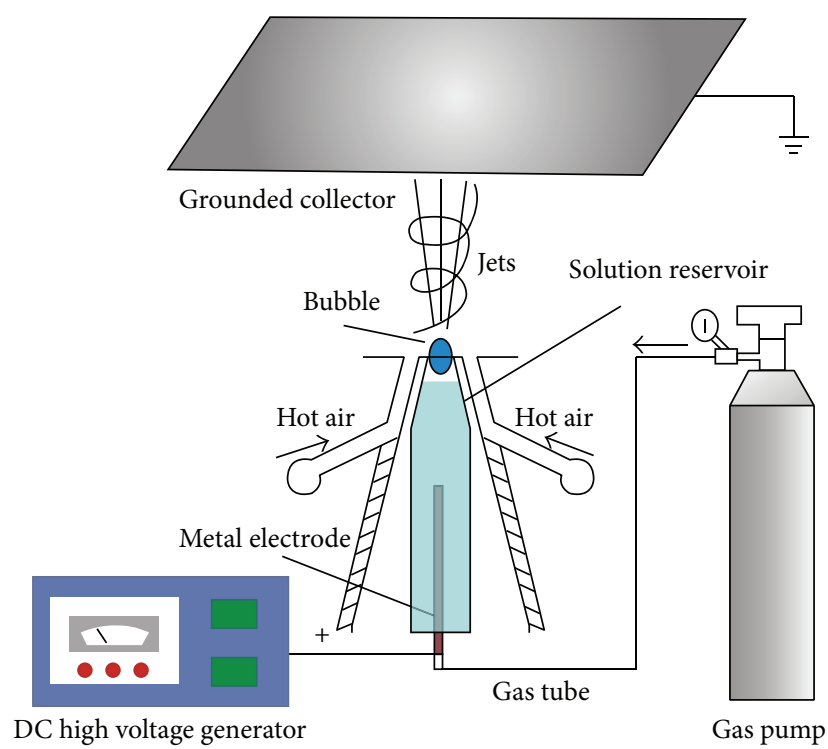

FIGURE 5: Air-jet assisted bubble electrospinning or electrostaticfield-assisted blown bubble spinning [14].

spinning, the system can alleviate safety-related concerns. In addition, centrifugal spinning can remarkably improve the production efficiency by increasing the rotational speed, allowing the fast and large-scale fabrication of nanofibers. Compared with the electrospinning process, it also allows nanofiber fabrication from the polymers at much higher concentrations, which decreases the use of solvent and lowers the production cost [20].

During a typical spinning process, a polymer solution is introduced into a highly rotating spinneret and then ejected as jets at a high rotating speed which is used to overcome the surface tension. The jets are then deposited on the collectors to form dried nanofibers as the solvent evaporated during the stretching process. Figure 7 shows a typical setup for centrifugal spinning [20].

Hammami et al. prepared nanoscale fibers using centrifugal force spinning and found that centrifugal force spinning also required certain chain overlap or entanglement. However, needles with smaller inner diameters were used for the centrifugal force spinning, which significantly improved the spinnability of the nanofiber [21].

Erickson et al. fabricated highly aligned chitosan/polycaprolactone $(\mathrm{PCL})$ nanofibers via centrifugal electrospinning system. Compared with those produced by the conventional electrospinning, the nanofibers produced by centrifugal electrospinning showed more uniform diameters and were aligned vertically. The diameter distribution of the nanofibers produced by centrifugal electrospinning was from $100 \mathrm{~nm}$ to $275 \mathrm{~nm}$, which was much narrower than that of the nanofibers produced by the conventional electrospinning $(25-450 \mathrm{~nm})$ [22].

2.4. Freeze-Drying. Freeze-drying (FD) can fabricate porous structures with controllable sizes directly from polymers, such as chitin, without structure-directing additives or pretreatments needed, which cannot be achieved by other techniques, such as self-assembly and electrospinning [23]. In addition, no high temperature and further leaching step are required for the freeze-drying process [24]. Therefore, it has drawn increasing attention in the fabrication of nanofibers. Starting with solution, emulsion, or dispersion, freezing leads the solute or solids to be excluded by ice front in the interstitial spaces between ice crystals. Then porous structures are created by the following sublimation [23].

$\mathrm{Ma}$ et al. prepared chitosan (CS)/sodium hyaluronate (SH) polyelectrolyte complex (PEC) fibers by FD method. The SEM images indicate that the equimolar ratio of CS to SH benefits the formation most. The in vitro tests indicate that the freeze-dried fibers have no cytotoxicity and good compatibility, which are essential for its application in aqueous systems, especially for biological and environmental applications [25].

$\mathrm{Wu}$ and Meredith prepared nondirectional and aperiodic chitin nanofibers (CNFs) by FD method. The prepared structures successfully mimicked the size and interconnectivity of white beetle structure with improved porosity. In all, the fine and porous structure can be realized by more facile waterbased FD method [23].

\section{Renewable Polymers for the Preparation of Nanofibers}

3.1. Chitosan. Chitosan (CS) is a natural cationic polyelectrolyte copolymer that can be obtained by the deacetylation of its parent polymer chitin, a naturally occurring source derived from the exoskeleton of insects, crustaceans, and certain fungi. The abundance as a renewable resource and excellent properties, such as hemostasis, biodegradability, biocompatibility, nontoxicity, and chelation with metals, render chitosan suitable for biomedical applications. Moreover, chitosan has also shown good performances in inhibiting the growth of a wide variety of yeasts, fungi, and bacteria as 


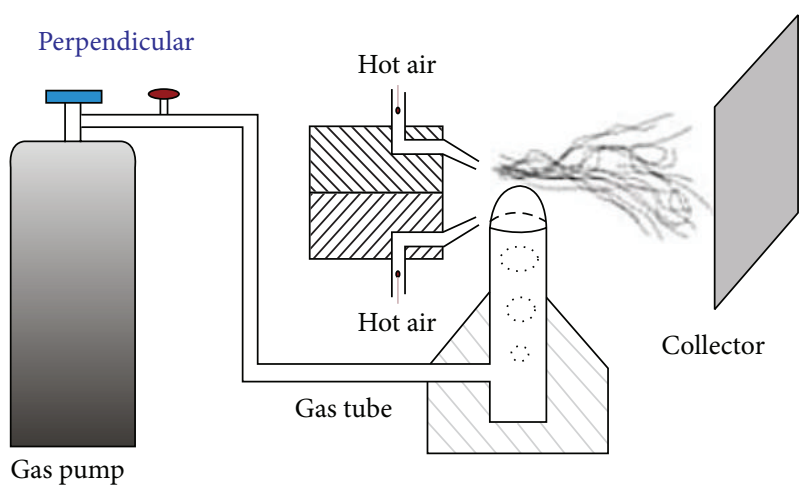

(a) Air flow direction is perpendicular to the path of the bubble ejection

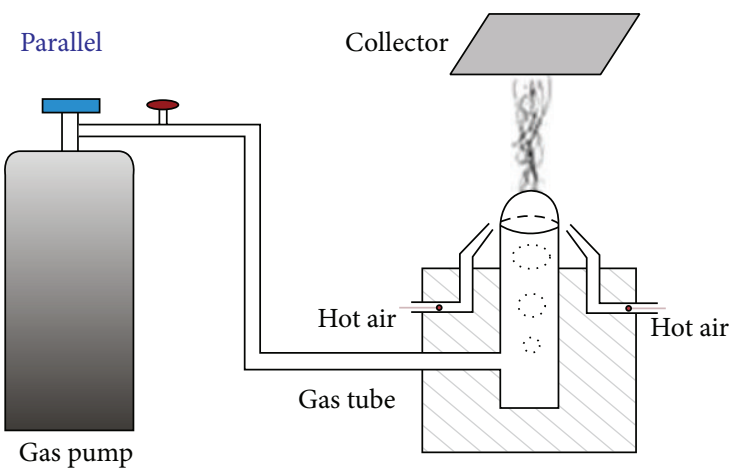

(b) Air flow direction is parallel to the path of the bubble ejection

Figure 6: The schematic of the experimental setup of two airflow directions: (a) perpendicular flow and (b) parallel flow [16].

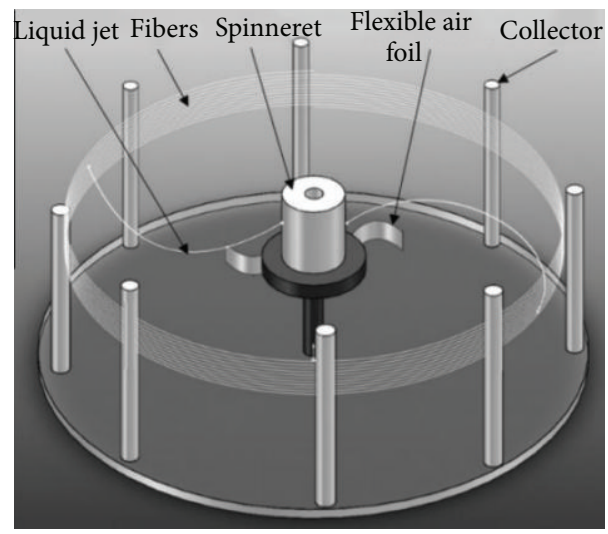

FIGURE 7: Setup of centrifugal spinning [20].

well as gas and aroma barrier properties in dry condition. All these positive features of chitosan have provided ample opportunities for further development in biomedical and other industrial fields [26-28].

It has been reported that pure chitosan fibers can be produced in nonaqueous solvents. For example, electrospinning of pure chitosan was successfully achieved in trifluoroacetic acid (TFA) by Ohkawa et al. [29]. Sencadas et al. improved the homogeneity of the nanofibers by adding dichloromethane to the chitosan/TFA mixtures and systematically studied the processing parameters to set the ground for a reproducible way to obtain chitosan nanofibers for specific applications [30].

However, electrospinning individual chitosan from its aqueous solution remains a challenge. First, the rigid Dglucosamine repeat unit, high crystallinity, and ability to form strong hydrogen bond of chitosan make it difficult to be dissolved in most solvents. In addition, its polycationic property also leads to high viscosity, which hinders the formation of sufficient chain entanglements and results in the formation of nanobeads rather than nanofibers [26, 31]. However, the solvents for the spinning of the pure chitosan are environmentally harmful [32]. The electrospinning for chitosan can only be achieved at concentrations in the range between $2 \%$ and $8 \%(\mathrm{wt} / \mathrm{v})$. This urges us to develop new versatile methods for the electrospinning of chitosan at higher concentrations to ensure the mechanical stability and retain the bioactivity of its nanofiber products [33].

Therefore, special attention needs to be paid to improving the spinnability of chitosan. Attempts have been made by blending chitosan with synthesized polymers, such as polyethylene oxide (PEO), polyvinyl alcohol (PVA), and PLA, and renewable polymers such as gelatin, alginate, and silk fibroin and nanoparticles to improve the mechanical strength, antibacterial activity, and antiadhesive properties towards bacteria of its nanofibers [31,34].

PVA is a typical nontoxic and water-soluble synthesized polymer with high biocompatibility and hydrophilicity. More importantly, PVA has good mechanical properties [35]. Liu et al. explored the preparation of PVA/chitosan hydrogel nanofibers by solution blowing method with ethylene glycol diglycidyl ether (EGDE) as cross-linker at various concentrations (as shown in Figure 8) and found that CS/PVA hydrogel nanofiber mats possessed the properties of both hydrogel and nanofiber mats. In addition to the similar excellent exudate absorption property to that of hydrogel films, hydrogel nanofiber mats allowed gaseous exchange, which decreased with the increase of its cross-linking degree (DCL). Moreover, CS/PVA hydrogel nanofiber mats demonstrated good antibacterial rates of over $81 \%$ against $E$. coli, which was not significantly affected by DCL. The findings suggest that the CS/PVA nanofiber hydrogel is a promising material for wound dressing [36].

Compared with synthetic polymers, natural polymers have better biocompatibility and lower immunogenicity. Sericin is one of the biodegradable and biocompatible natural polymers with good antioxidant, moisture absorption, and antibacterial and UV resistance properties and has been widely used in cosmetics and fabrics. Zhao et al. prepared chitosan/sericin composite nanofibers by blending binatural polymers and electrospinning to enhance their biological performance. The nanofibers had diameters ranging from $240 \mathrm{~nm}$ to $380 \mathrm{~nm}$ and continuous and uniform diameter distribution. Bioassays indicated that these novel composite nanofibers showed noncytotoxicity and remarkably 


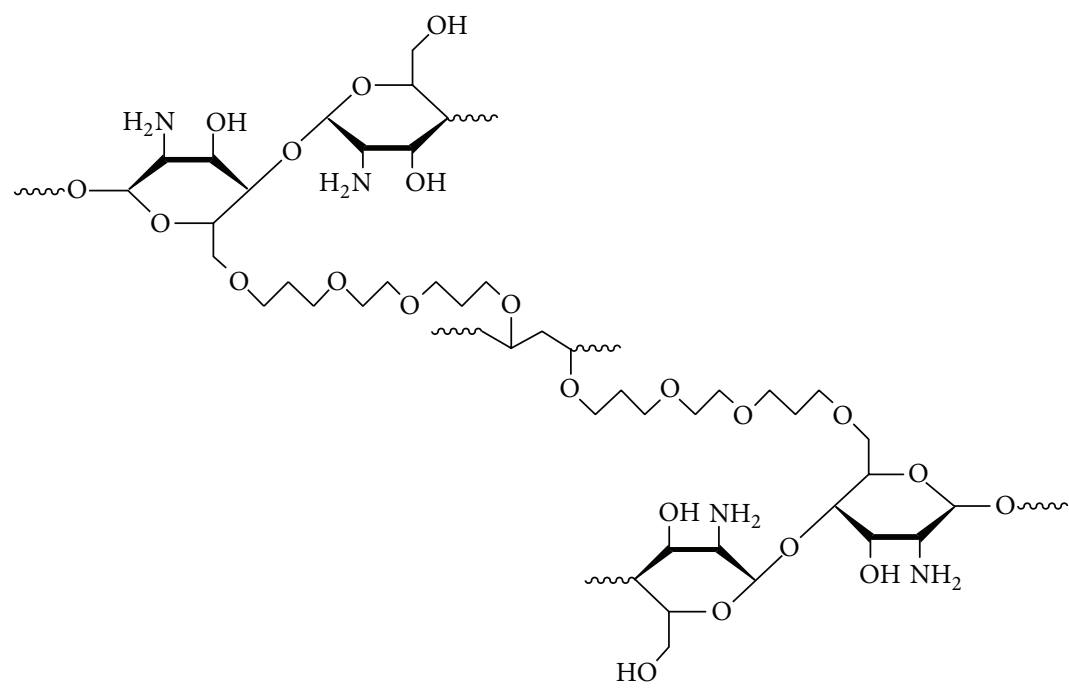

FIGURE 8: The structure of CS/PVA hydrogel nanofibers cross-linked by EGDE.

enhanced cell proliferation and antibacterial activities against both Gram-negative bacteria Escherichia coli and Grampositive bacteria Bacillus subtilis [34].

Ag nanoparticles (Ag-NPs) have shown superior antibacterial properties. Ag-NPs can attach to cells to interrupt their permeability and intermembrane exchange. In addition, AgNPs have no adverse effects on viable cells and cause no significant microbial resistance. Therefore, Ag-NPs have been widely used as additives to various materials and in wound dressings against microbial contaminations [35, 37]. Abdelgawad et al. successfully prepared chitosan/PVA/Ag-NPs nanofiber mats with chitosan as capping polymer at high concentrations and glucose as reducing agent for antimicrobial applications. Ag-NPs were able to improve the electrospinnability of the blend, reduce the diameter of fibers, and more importantly enhance the antibacterial performances of the electrospun fibers, which made them viable to wound dressing [37].

3.2. Cellulose. Cellulose is one of the most abundant biodegradable materials and can be extracted from different natural sources, such as wood and cotton pulps, via chemical and/or physical methods [38].

Bacterial cellulose (BC) is natural cellulose synthesized by acetobacter xylinum [38] and has high biocompatibility and transparency, nontoxicity, and excellent mechanical strength. Moreover, BC fibers have a very high surface area per unit mass, which, combining with its high hydrophilicity, leads to a very high liquid loading capacity. Thus, BC has been applied in various fields [39]. Costa et al. carried out electrospinning of acetylated BC nanofiber mats to produce artificial symmetric nanoporous structures. The SEM images revealed that nanopores were more uniformly distributed throughout the electrospun nanofiber mat than those casted with BC mats. In addition, the electrospinning of modified BC nanofibers was easier than that of unmodified BC nanofibers, which could be explained by the dissolution mechanism of cellulose in DMA/LiCl solvent system [40].
However, BC shows poor antibacterial, antioxidant, and conducting and magnetic properties, which limits its application in biomedical and electronic fields [41]. One of the solutions is to blend $\mathrm{BC}$ with other substances to promote its properties. Carbon nanotubes (CNTs) have been of great interest in various fields since their discovery. They are promising fillers for polymer matrices to prepare CNTsreinforced composite materials because of their extraordinarily high aspect ratio, elastic modulus, and high axial strength. Chen et al. prepared multiwalled CNTs-embedded BC nanofibers (MWCNTs/BC nanofibers) by electrospinning. The TGA analysis showed that the initial temperature of degradation followed the increasing order of $\mathrm{BC}<$ electrospun BC nanofibers < electrospun MWCNTs/BC nanofibers, which was attributed to the crystalline polymorph transformation and orientation as well as the embedded MWCNTs. In addition, the mechanical properties including tensile strength and Young's modulus and electrical conductivity of the products were significantly enhanced due to the welldispersed and aligned MWCNTs. The finding indicates that MWCNTs/BC nanofibers may be potentially used in medical, mechanical, and electrical fields [42].

Cellulose acetate (CA) is a derivative of cellulose and has good stability and solubility in organic solvent. It can also selectively absorb low-level organic compounds and toxins. However, CA has poor mechanical properties [43]. Khalf et al. fabricated a CA/polycaprolactone (PCL) core-sheath nanostructure by coaxial electrospinning to promote its mechanical and biological properties. The preparation process is shown in Figure 9. Their results indicate that the loading of PCL was able to improve the elastic elongation of CA solid and hollow fibers. Cell attachment test suggested that the nontoxicity and higher cell attachment performance of CA nanofibers blended with PCL were attributed to its higher surface area per volume ratio [44].

3.3. Alginate. Alginate is a natural linear polysaccharide copolymer and can be obtained from brown sea weed. 


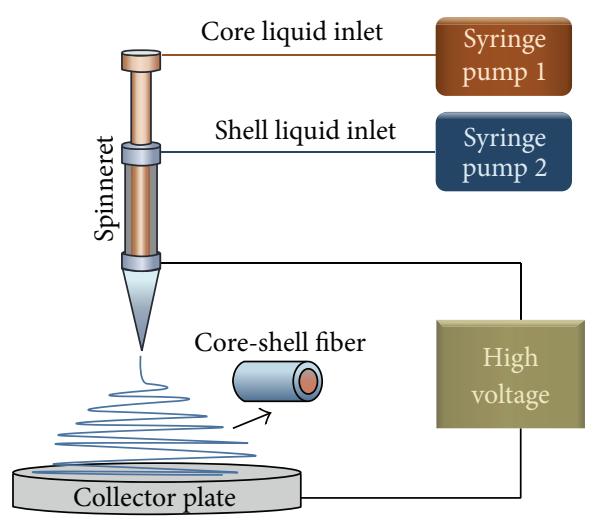

(a)

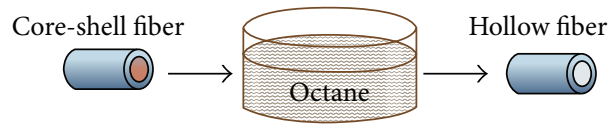

(b)

FIGURE 9: The preparation process of CA/PCL core-sheath nanostructures [44].

Sodium alginate (SA) has been well studied on its biomedical applications due to its superior properties including biocompatibility, biodegradability, nontoxicity, hydrophilicity, and low cost. Unlike chitosan, alginate is highly soluble in water [45].

To improve the structural integrity of the prepared nanofibers, alginate-based materials are usually cross-linked with divalent ions, such as calcium and barium. In addition, double cross-linking with glutaraldehyde, hexamethylene diisocyanate (HMDI), epichlorohydrin, and adipic acid (ADA) hydrazide has also been proposed (as shown in Figure 10). Unlike the non-cross-linked electrospun nanofibers, the cross-linked electrospun nanofibers are highly stable and relatively insoluble in both water and simulated body fluid [46-48].

However, pure SA nanofibers have been rarely fabricated via electrospinning. Nie et al. [49] prepared smooth and uniform alginate nanofibers by introducing glycerol as a cosolvent and simply adjusting the volume ratio between glycerol and water. Fang et al. [50] reported that pure alginate had been successfully electrospun by introducing $\mathrm{Ca}^{2+}$ cations to SA aqueous solutions. They demonstrated that high intermolecular interactions and low surface tension were the critical factors to improve the electrospinnability of SA solutions.

Alginate has poor properties including low mechanical strength, high degradation rates, and processing difficulties, which are usually overcome by compositing alginate with other polymers such as PEO [51] and PVA [45]. It can be attributed to the intermolecular hydrogen bonds formed between the polymers and alginate. Surfactants, such as lecithin [52], and cosolvents have also been proposed to facilitate its processing for the preparation of nanofibers [53].

3.4. Gelatin. Gelatin (GT) is a renewable polymer obtained by the partial hydrolysis of collagen, a most abundant natural substance in connective tissues. GT has been widely used as a bioengineering material due to its high biocompatibility, biodegradability, and low cost. GT can also prevent fluid loss, which plays an important role in wound healing [54-56]. However, the electrospun gelatin nanofibers are soluble in water and have poor mechanical strength $[56,57]$. Therefore, cross-linking treatments have been adopted to increase their water resistance [13] and mechanical properties [58, 59]. The cross-linking can be realized by physical processes, such as drying, heating, $\gamma$-ray, electron beam, and UV light exposure and chemical treatments by using cross-linker, such as formaldehyde, glutaraldehyde (GA), epoxy compounds, tannic acid, carbodiimide, acyl azide, and transglutaminase [60], through dissolving gelatin in 2,2,2-trifluoroethanol [61], formic acid dope solution [62], and water-based cosolvent composed of ethyl acetate and acetic acid [63]. Among all chemicals, GA is by far the most widely used due to its high efficiency, easy accessibility, and low cost. However, glutaraldehyde at high concentrations is cytotoxic and can disrupt the electrospun fiber morphology [59, 64]. Ko et al. [60] demonstrated that the gelatin nanofibers cross-linked with $0.5 \%(\mathrm{w} / \mathrm{v})$ genipin promoted cell proliferation and reduced its solubility and cytotoxicity. Lu et al. [65] fabricated gelatin nanofibers by electrospinning and compared the properties of the gelatin nanofibers cross-linked by vapor and liquid-phase GA. Their results indicated that the crosslinking by both vapor-phase and liquid-phase GA was able to improve the mechanical strength of the gelatin fibers. The cross-linking with liquid-phase GA resulted in more evenly cross-linked, close-packed, and higher tensile. The excessive cross-linking with vapor-phase GA led to membranes shrinking due to poorly cross-linked middle layers in their hierarchy structure. Therefore, a novel in situ cross-linking method was proposed by Slemming-Adamsen et al. [66] for the fabrication of electrospun gelatin nanofibers with 1-ethyl3-(3-dimethylaminopropyl)-1-carbodiimide hydrochloride (EDC) and N-hydroxysuccinimide (NHS) as cross-linkers. Kwak et al. [67] prepared Phaeodactylum tricornutum ( $P$. tricornutum) extract-loaded gelatin nanofibers via electrospinning. $P$. tricornutum is a diatom which exists in brackish and marine water worldwide. The water-soluble extracts of P. tricornutum have showed anti-inflammatory, analgesic, and free radical scavenging activities. The introduction of $P$. tricornutum extracts improved the conductivity of the gelatin solution and reduced the diameter of the nanofibers. Their results also confirmed the antimicrobial activity and noncytotoxicity of the P. tricornutum-loaded gelatin nanofibers.

Gelatin has also been used as a blend component to prepare nanofibrous membranes for tissue scaffolds, wound healing and health caring devices, and other biomedical applications. Shan et al. [68] prepared silk fibroin/gelatin (SF/GT) electrospun nanofibrous dressing loaded with astragaloside IV (AS), an effective component, to accelerate wound healing. They found that the nanofibers were able to significantly improve cell adhesion and proliferation, promote wound healing, induce proangiogenesis of partial thickness burn wound, and inhibit complications in wounds. In addition, SF significantly decreased the average diameter, 


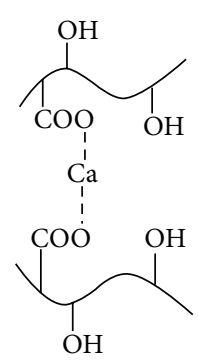

(a)

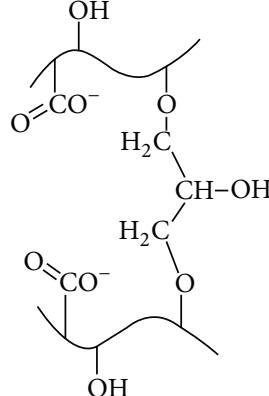

(b)

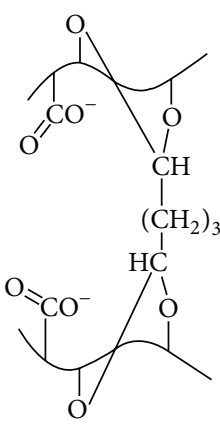

(c)

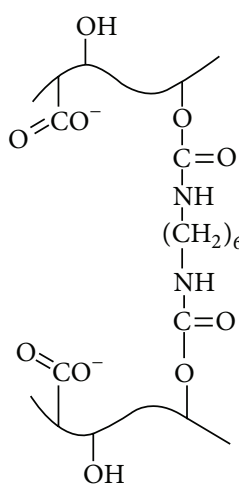

(d)

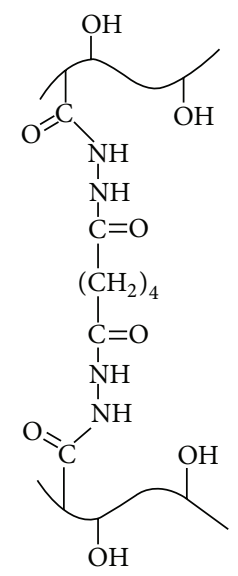

(e)

FIGURE 10: Molecular network structures formed between alginate chains in nanofibers after cross-linking with (a) $\mathrm{CaCl}_{2}$, (b) epichlorohydrin, (c) glutaraldehyde, (d) HMDI, and (e) ADA [46].

degradation rate, and the facture strain under high break strength of the produced nanofibers.

In addition to the general properties of a condensed polyphenol, the grape seed polyphenol (GSP) has some unique properties, such as antimicrobial activity, anti-inflammatory activity, and reducing blood sugar and fat. Han et al. [69] fabricated GSP/gelatin composite nanofibers containing Ag-NPs by electrospinning as a novel biomaterial. The process is shown in Figure 11. GSP served as the reducing agent and the stabilizing agent for silver nanoparticles. The electrospun composite nanofibers had average diameters in the range of 150-230 $\mathrm{nm}$ and a uniform distribution of Ag-NPs with an average diameter of $11 \mathrm{~nm}$ that contributed to their good antibacterial performance. Further investigation also revealed that the composite nanofibers containing silver nanoparticles had no toxicity at such concentrations. Thus, they could be potentially utilized in biomedical field as a wound dressing.

3.5. Hyaluronic Acid. Hyaluronic acid (HA) is one main glycosaminoglycan which is found in the ECM of many soft tissues of higher animals [70]. HA can decrease the formation of postsurgical adhesions due to its noncoagulant activity, modulation of fibrous tissue, barrier effect on fibrinogen, and inhibitory effect on the superoxide release from granulocytes [71]. Previous studies have demonstrated that HA nanofiber wound dressing showed better performance than the solid $\mathrm{HA}$ in wound healing due to its unique morphologies. However, HA solutions have high solution viscosity even at low concentrations, leading to issues in the production of consistent nanofibers [72].

Brenner et al. prepared pure HA nanofibers by electrospinning in a solvent system of aqueous ammonium hydroxide $\left(25 \% \mathrm{NH}_{4} \mathrm{OH}\right)$ and $\mathrm{DMF}$ at $\mathrm{pH}$ of 11 to eliminate the solvent induced degradation effects on the biopolymer as reported in the previous studies. Continuous, cylindrical, and randomly oriented pure HA nanofibers with a diameter of $39 \pm 12 \mathrm{~nm}$ and good biocompatibility were successfully electrospun [70].
Uppal et al. fabricated HA nanofibers by electrospinning with deionized water as a solvent and cocamidopropyl betaine as a surfactant. Based on the report of histopathologist, the sterilized HA nanofibers wound dressing showed better pathology performance than other sterilized wound dressings including solid HA, gauze with Vaseline dressing, adhesive bandage, and antibiotic wound dressing. The evaluation criteria included epithelial tissue gap, epithelial tissue detachment from the dermis, the presence of granulation tissue, and the assessment from clinic photographs based on the color of the wound and whether the wound was fully covered or not by the epithelial tissue. In addition, it showed higher air permeability than sterilized solid HA and gauze with Vaseline dressing, which contributed to the faster wound healing [72]. In all, the addition of antibiotic components could lead to better performance of HA nanofibers.

Chen et al. prepared dual functional core-sheath structured nanofibers with HA as the inner core and Ag-NPsloaded PCL as extremal sheath by coaxial electrospinning. This design was used to control the slow release of HA from the core to mimic the biological function of HA in synovial fluid containing high concentration of HA. The sheath acted as a barrier with antimicrobial activity to prevent infection and alleviate adhesion. The results demonstrated that the core-sheath structure met the need of preventing peritendinous adhesion and postoperative infection through the slowly released HA and rapid release of Ag-NPs without exhibiting significant cytotoxicity [71].

3.6. Collagen. Collagen is the principal structural element of the native ECM in a three-dimensional network structure composed of multifibrils in a nanofiber scale. Due to a wealth of merits including biological origin, nonimmunogenicity, and excellent biocompatibility and biodegradability, collagen has been widely used as biomaterials in pharmaceutical and medical fields such as carriers for drug delivery, dressings for wound healing, and tissue engineering scaffold. Among all isoforms of collagen, the fibrillar structure of type I 


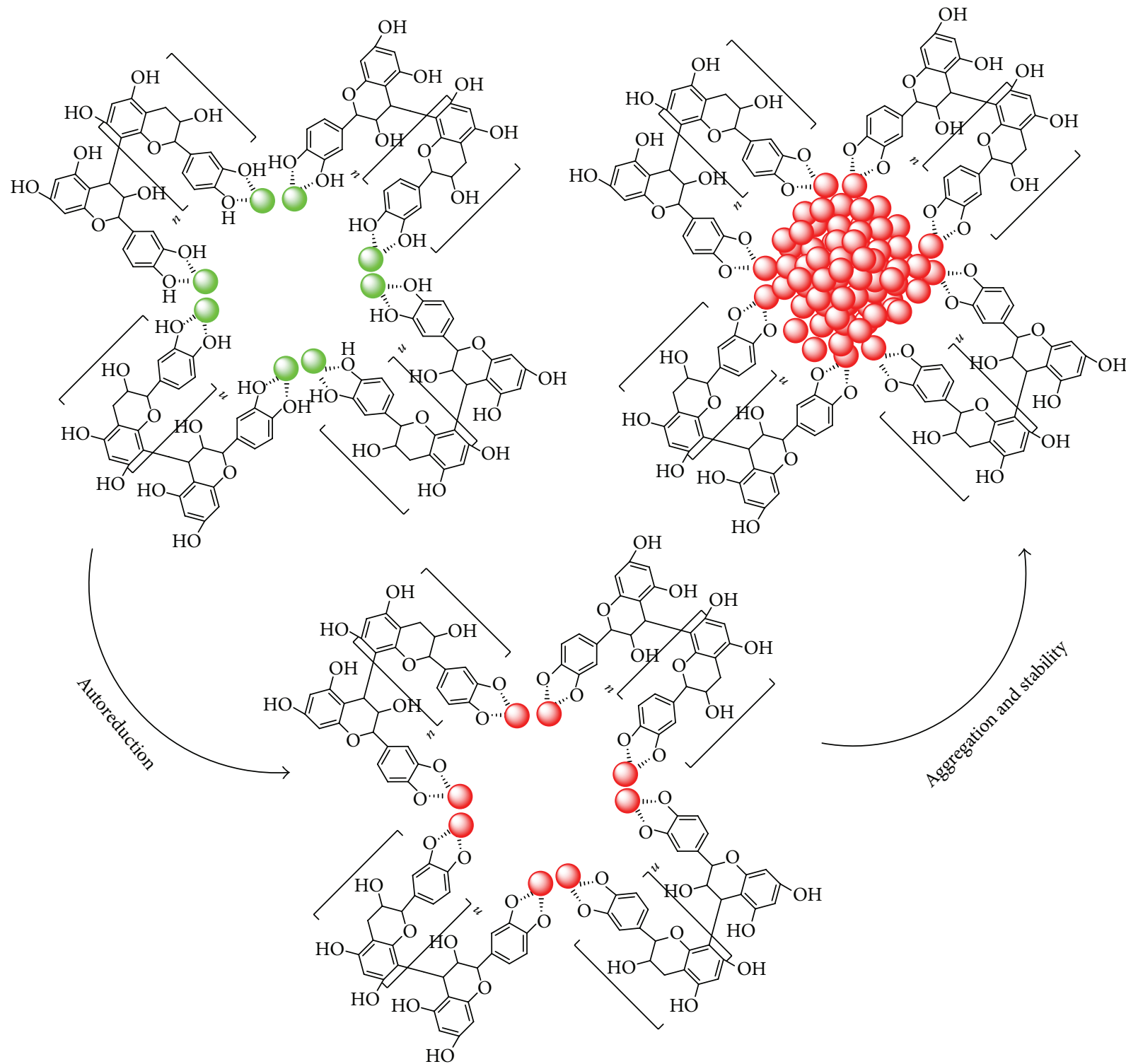

$\mathrm{Ag}^{+}$

$\mathrm{Ag}^{0}$

FIGURE 11: Schematic representation of the mechanism for preparing silver nanoparticles with GSP [69].

collagen has long been known for its important roles in cell attachment, proliferation, and differentiation [73, 74].

The adaptations of electrospinning to produce tissue engineering scaffolds composed of collagen nanofibers have shown that the structural properties of the electrospun collagen vary with the tissue of origin, the isoform, and the concentration of the collagen solution [75].

Collagen-composite fibrous mats have been fabricated with PEO [76], PCL [77, 78], polylactide-polyglycolide (PLGA) [75], poly(L-lactide-co-e-caprolactone) (PLCL) [79], chitosan [73], hydroxyapatite [80, 81], and poly(L-lactic acid)co-poly( $\varepsilon$-caprolactone) (P(LLA-CL)) [82]. Chen et al. [83] prepared the composite nanofibrous membrane (NFM) of type I collagen, chitosan, and PEO by electrospinning, which was further cross-linked with GA vapor. The cross-linking increased Young's modulus of the NFMs and decreased their ultimate tensile strength, tensile strain, and water sorption capability. The NFMs showed no cytotoxicity toward the growth of 3T3 fibroblasts and demonstrated good biocompatibility in vitro. In addition, the NFMs showed better wound healing performances than gauze and commercial collagen sponge wound dressings. This novel electrospun matrix can also be potentially used as a wound dressing for skin regeneration. Choi et al. [78] examined the feasibility of using PCL/collagen based nanofibers as a scaffold system for implantable engineered muscle organization. Their results 
showed that, in contrast to the randomly oriented nanofibers, unidirectionally oriented nanofibers could induce muscle cell alignment and myotube formation. The aligned composite nanofiber scaffolds seeded with skeletal muscle cells may be able to provide implantable functional muscle tissues. Liu et al. [84] explored the incorporation of neurotrophin (NT3 ) and chondroitinase $\mathrm{ABC}$ (ChABC) into the electrospun collagen nanofiber for spinal cord injuries (SCI) treatment. The sustained release of NT-3 and ChABC for at least 28 days was achieved. Such biofunctional scaffolds may be found useful in SCI treatment by providing topographical and multiple biochemical cues to manipulate the growth inhibitory environment and promote axonal regeneration.

3.7. Silk Fibroin. Silk fibroin (SF) is a main component of silk. It is a type of fibrous proteins with remarkable mechanical properties produced in the form of fiber by silkworms and spiders. SF is also a promising wound dressing material due to its excellent mechanical properties, as well as its biocompatibility, controllable biodegradability, water-based processing, morphologic flexibility, and easily accessible chemical groups for functional modification. Despite its advantages, the low availability of SF limits the industrial scale production of its biomaterials. Generally, the electrospun SF fibers with desirable properties for bioengineering are prepared with $\mathrm{SF}$ at very high concentrations in the range of $20-40 \%$. In addition, the highly crystalline $\beta$-sheet secondary structures result in the insolubility of SF, which limits its biomedical applications. Therefore, electrospun SF nanofibers are usually chemically treated with methanol [85], ethanol, propanol [86], and water vapor [87] to increase their stability. Strategies have been proposed to increase the viscoelasticity of SF systems by blending them with other polymers, which improves the mechanical properties while maintaining the biocompatibility of the obtained nanofibers [88-91].

Calamak et al. [92] fabricated silk fibroin nanofibers containing UV-reduced Ag-NPs by electrospinning method and posttreated the product with methanol and GA vapor. The posttreatments promoted the mechanical properties of the SF bionanotextiles. The results indicate that the methanol treatment was more effective in the crystallization transition of fibroin from random coil to $\beta$-sheet. In addition, Ag-NPs significantly decreased the diameter of the nanofibers and contributed to their antibacterial activity against Gram-positive bacteria $S$. aureus and Gram-negative bacteria P. aeruginosa.

Polyethylenimine (PEI) is a polycationic antimicrobial polymer with interesting ability to enter cells or permeabilize cell membranes. Çalamak et al. [90] prepared silk fibroin based antibacterial bionanotextiles and explored their application as wound dressing materials. The addition of PEI to the nanofibers decreased the average diameter of the nanofibers and increased their hydrophobicity, cell viability, and antimicrobial activity against both Gram-positive and Gram-negative bacteria. In contrast, the sulfated SF only showed antibacterial activity against $P$. aeruginosa.

Shahverdi et al. [89] fabricated silk fibroin blended with PLGA nanofibers via a dual source electrospinning setup, through which the electrospinning of SF and PLGA nanofibers could be separately optimized and achieved. They found that the concentrations of SF and PLGA predominantly affected the formation of the bead structure in the fibers. The addition of PLGA significantly improved the mechanical properties of the nanofibers. The incorporation of SF with PLGA displayed better cell attachment, proliferation, and viability performance than pure SF nanofibers and pure PLGA nanofibers.

A novel tissue engineering scaffold of electrospun silk fibroin/nanohydroxyapatite (nHA) biocomposite was prepared by an effective calcium and phosphate $(\mathrm{Ca}-\mathrm{P})$ alternate soaking method. The cell cultivation experiments demonstrated that the silk/nHA nanocomposite scaffold could support the early stage of osteoblast adhesion and had a significant effect on the differentiation stage. Their findings suggest that this composite scaffold can be a promising biomaterial for bone tissue engineering [93].

$\mathrm{Ki}$ et al. [94] determined the characteristics and performance of an electrospun membrane prepared with wool keratose (WK) and SF protein for metal ion absorption. The nanofibrous membranes exhibited higher metal ion absorption capacities than the conventional fibrous filters (wool sliver and filter paper). Particularly, the WK/SF blend nanofibrous membrane showed an extraordinary performance for the absorption of metal ions. In addition, its absorption capacity remained stable after several adsorption-desorption cycles. The electrospun WK/SF nanofibrous membrane is suitable for removing and recovering heavy metal ions in water.

\section{Applications of Renewable Polymer Based Fibers in Wound Dressing}

Effective wound dressings are highly desired in various areas, such as promoting wound healing, hemostasis, skin regeneration, diabetic ulcers, and tissue engineering. A good wound dressing can maintain a moist environment at the interface, provide mechanical protection, prevent bacterial penetration, and allow gas-fluid exchange. In addition, it is nonadhesive to the wound and easily removed without trauma. However, the dressings used in specific fields may have their unique properties and requirements.

4.1. Promoting Wound Healing. A variety of fibers prepared with renewable materials including chitosan, fibrinogen, silk fibrin, bacterial cellulose, gelatin, and collagen and composites of these materials can promote wound healing and show antibacterial activity, good adhesion, good absorption, and nontoxicity and sufficient gas-exchange properties. The mats prepared with the fibers have unique porous structures and high surface areas, which are beneficial to cell regeneration and the wetness of the wound. In addition, the mats can protect the wound from the harmful external environments [95].

The bioactive molecules or drugs can be easily incorporated in the fibers by electrospinning to provide additional functions such as anti-inflammatory activity and tissue growth-promoting activity $[96,97]$. Wu developed a composite dressing of alginate, hydroxyethyl cellulose, and growth factor and successfully applied it to the treatment of facial wounds [98]. 
Chitosan fibers blended with other polymers, such as PEO, PLA, PVA, and PCL, have been proved as promising candidates for wound healing dressing [99]. Huang et al. reported that chitosan/poly-L-lactic acid (PLLA) composite nanofiber prepared by electrospinning showed better performance in maintaining wettability than pure PLLA and thus accelerated wound healing [100]. Theisen et al. found that blending gelatin with PLLA led to a better performance for cell proliferation and ECM production and the cells were able to grow into tissue-like constructs [101]. Alginate might provide gelation and moist healing environment for chronic wounds, boosting their reepithelialization [102]. de Moraes and Beppu prepared a biocomposite membrane with sodium alginate and silk fibroin fibers. The incorporation of fibroin fibers enhanced the tensile strength of the fibers and resulted in the properties of a typical biomaterial for biomedical applications such as wound dressing. Its nontoxicity and flexibility also contributed to the fast wound healing [103].

4.2. Hemostasis. Timely and rapid hemostasis is very important to avoid further complications in surgical operation and emergency medical treatment. An effective hemostatic dressing should have properties such as excellent hemostasis, good biocompatibility, nontoxicity, no antigenicity, low cost, and easy processing [104].

Various renewable polymers, such as fibrinogen, fibrin, alginate, chitosan, cellulose, and gelatin, have been used in the preparation of hemostatic dressings. These materials in the form of fiber have shown excellent performances in hemostasis due to the large contact areas with blood which promotes the aggregation and adhesion of thrombocyte on the fibers. In addition, the soft and strong fiber can closely adhere to the wound and press the wound to promote hemostasis.

Zhao et al. prepared a nanofiber of fibrinogen fiber by electrospinning and applied it to hemostasis. The clinical study indicates that the dressing was able to significantly shorten bleeding time and decrease bleeding volume of splenic hemorrhage, hepatic hemorrhage, and femoral arteriovenous fistula, thus reducing mortality rate [105]. The fibrinogen forms a tough fibrin clots once it is activated by thrombin. The clot adheres to the wound and promotes hemostasis to accelerate wound healing. In addition, cross-linked fibrinogens have certain toughness and viscosity, which can prevent blood flushing and provide a physical hemostasis [106].

Fibrin is used in hemostasis due to its biocompatibility and excellent biological properties [107]. Fibrin is an initiator for the regulation of thrombosis and hemostasis. It is decomposed by fibrinogen, the major component of plasma proteins, when human body gets wounded and immediately covers the wound to accelerate the hemostasis [108]. During the healing process, fibrin can be completely degraded and absorbed by the tissue and causes no damage to the organism nearby.

Alginate is also an excellent hemostatic material. Pillai and Naieb prepared a ventral nonadhesive dressing with a soft net of pure calcium alginate, which showed an excellent hydrophilism. During the treatment, it swelled upon the absorption of exudate and formed a firm gel filled wound without adhesion, which maintained a moist environment for the wound and promoted its healing [109]. In addition, $\mathrm{Ca}^{2+}$ in the alginate fibers could be transferred into the blood by exchanging with $\mathrm{Na}^{+}$and acted as a coagulation factor to accelerate the hemostasis.

Sibaja et al. synthesized a fiber by composing chitosan with alginate through wet extrusion and found that it was a biocompatible material with acceptable tensile properties for the inhibition of body fluids, such as blood, from flowing. The positive $-\mathrm{NH}_{3}{ }^{+}$groups on the skeleton of chitosan can interact with the negative charges on the surface of the red blood cells and platelets, accelerate their aggregation, and promote the hemostasis. Studies have also shown that the incorporation of chitosan into alginate fibers can significantly enhance the tensile properties of the dressing [110].

Liu et al. prepared several novel nonwoven hemostatic dressings composed of chitosan derivatives including carboxymethyl chitosan fiber, hydroxypropyl chitosan fiber, hydroxyethyl chitosan fiber, and carboxymethyl hydroxyethyl chitosan fiber. The dressing showed good biocompatibility, excellent tensile strength, and easy processing properties and had no antigenicity. Their study also demonstrated that the dressings promoted hemostasis and wound healing by forming a colloidal solution during the swelling and dissolving. Therefore, the dressings are especially suitable in the field of in vivo hemostasis [111].

Dressings prepared with cellulose have been widely applied in the surgical hemostasis because they can quickly stop bleeding and have a strong affinity for water and normal saline. The dressing absorbs water from blood and is dissolved to form a gel that plugs the capillary end to increase the viscosity of the blood and decelerate blood and thus stops bleeding. The hemostasis of cellulose dressing is based on mechanical press, erythrocyte absorption, and thrombocyte aggregation. Wang et al. prepared a soluble hemostatic fiber of carboxymethyl cellulose, which showed an excellent hemostatic performance in the clinical experiments [112]. Compared with gelatin sponge, the dressing was able to more effectively control bleeding of small blood vessels during the surgery, especially the blood infiltration of wound and suture. It is believed that the carboxymethyl cellulose fiber can accelerate platelet adhesion and reduce postoperative hematoma.

Niu et al. blended gelatin with lidocaine and PVA to produce nonwoven hemostatic materials. The produced dressing had a thin and dense network structure with numerous loose gaps that increased the contact area for the blood and thus promoted thrombocytes aggregation and the formation of clot and accelerated hemostasis [113]. PVA is a high expansion material having a high degree of hydrophilicity, which makes the dressing expand quickly to partially press the wound and stanch the bleeding [114].

4.3. Skin Regeneration. Nowadays, burn healing is one of the most significant problems of modern surgery because of the high lethality and disability after the treatment for burns of large surface area. Patients with burn injuries undergo significant emotional and physical pain and trauma from the burn, dressing changes, and the related treatments during the healing process [115]. In addition, fluid loss, 


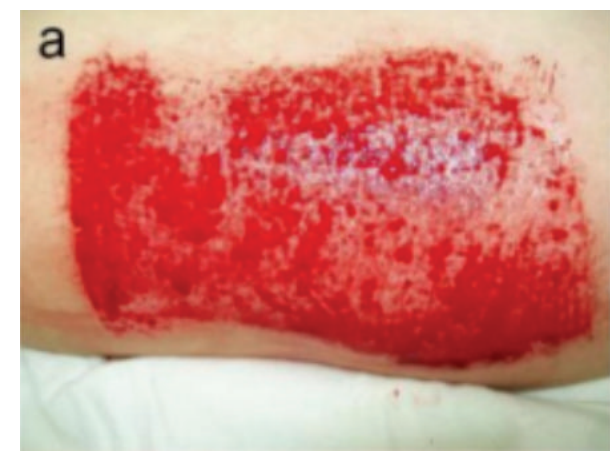

(a)

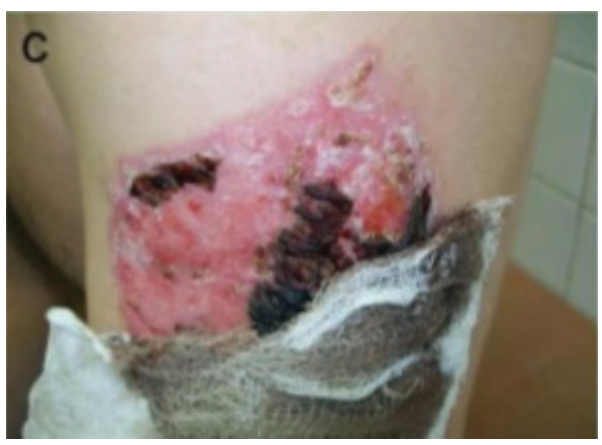

(c)

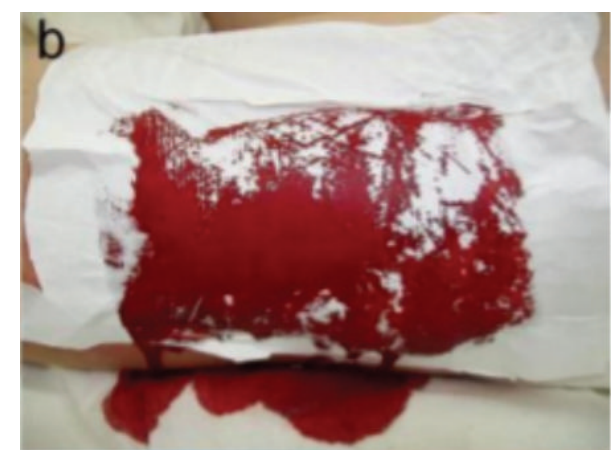

(b)

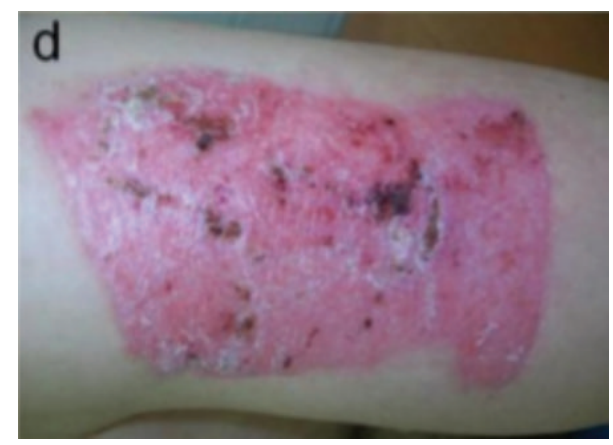

(d)

FIGURE 12: Donor wound: (a) before covering; (b) after covering; (c) 12 days after covering; and (d) 14 days after covering [121].

bacterial invasion, passive evaporative water, heat loss, and other complications can prevent wound from healing [116]. Therefore, the ideal burn dressing should have the following properties [117, 118]: (1) high exudate absorption capability, (2) ability to protect the wound from microorganisms, (3) being removable from the injured skin surface, (4) sufficient oxygen permeability, (5) high water vapor permeability, (6) nontoxicity, and (7) ability to accelerate scar formation. Covering large burned surfaces is still a challenge.

Fibrous wound dressing material is a promising candidate for skin regeneration. For example, Venugopal and Ramakrishna found that gelatin nanofibers could speed wound healing and could be used for cell transfer and skin regeneration [119]. Various blend fibers of renewable polymers, such as $\mathrm{PEO} /$ chitosan [120], PCL/collagen [119], PCL/gelatin [121], PLLA/chitosan [122], Zein/collagen [123], and PLLA/gelatin [124], are the dominate candidates.

The micro- and nanofibers of chitosan are an ideal candidate for burn dressing because of its high surface area to volume ratio [120]. The nanostructure of fibrous materials with small pore size functions as biological filters to effectively retain water and prevent infection from various bacteria. Kossovich et al. prepared a novel nanofiber dressing by blending chitosan with PEO through an electrospinning in glacial acetic acid. The clinical study indicates that the chitosan nanofiber dressing showed antibacterial properties and high absorption capacity and was able to effectively absorb exudate, ventilate wound, protect the wound from infection, and stimulate skin tissue regeneration. In addition, the chitosan-based dressing showed excellent biodegradation, which avoided the mechanical damage to the wound from the wound dressing removal, reduced pain from dressing removal and change, and fastened rehabilitation [120]. Figure 12 shows that the wound dressing applied to the donor wound of transplant skin removal promoted skin regeneration by keeping a moist environment and removing the surplus of exudate without dressing change.

Venugopal et al. reported that composite fiber of collagen and PCL could support the growths of human dermal fibroblast and keratinocytes in skin engineering and the PCK film could not. They believe that cells migrated through pores in the fibers by amoeboid movement, pushing the surrounding fibers aside to expand. The dynamic architecture may provide cells with an opportunity to adjust the optimal pore diameter and grow into the scaffold. Chaisiri et al. prepared a skin tissue scaffold with composite fibers of gelatin and PCL to mimic the natural dermal ECM. The scaffold had a pore size of $40-$ 70 micron and $98 \%-99 \%$ of porosity. In addition, it is biocompatible and can support cell migration and proliferation in its network [121].

4.4. Diabetic Ulcers. Diabetes mellitus is one of the most important public health problems worldwide because of its high prevalence and enormous social and economic consequences. It has been reported that there are more than 135 million diabetics in the world and the number is expected to increase to 300 million in the next 25 years [125]. 
Foot ulcers are a common, serious, and costly health issue for people with diabetes around the world. Two common chronic complications of diabetes mellitus including peripheral neuropathy and vascular insufficiency may lead to diabetic foot ulcers. Diabetic patients have a sharp decreased fibroblasts activity provoked by unavailability of glucose for aerobic metabolism, resulting in insufficient fibrous collagen tissue proliferation. Imbalance in these processes has been believed to be the main reason for the occurrence and persistence of this kind of disease. The wound healing process involves the interaction between various cellular groups, ECM, and growth factors, which is affected by the vascular insufficiency and severity of the lesion and infection.

Many renewable polymers, such as chitosan, alginate, cellulose, and silk, can be used to produce the dressings for the diabetic ulcers. Wang et al. developed a dressing composed of chitosan fibers, calcium alginate fibers, and chemically modified cellulose fibers and successfully used it to treat diabetic ulcers. In addition, epidermal growth factor (EGF) was incorporated into the renewable polymers to further promote the healing [126]. Schneider et al. prepared a silk mat with electrospun nanoscale silk fibers containing EGF, from which the EGF was slowly released in a time-dependent manner (25\% EGF released in $170 \mathrm{~h}$ ). Clinical study shows that the silk mat aided the healing by increasing time of wound closure by $90 \%$ by epidermal tongue, especially in chronic wounds including diabetic ulcers. Renewable polymers blended with metals can also show anti-inflammatory activity to accelerate the healing. Lázaro-Martínez reported a dressing of the blend of alginate fiber and hydrocolloid silver with excellent performances in decreasing inflammatory clinical signs and promoting healing on diabetic ulcers. The clinical study indicates that it reduced wound area with a median percentage of $47.7 \%$ and a mean percentage on the lesion of $58 \%$ at 2 weeks and $67.14 \%$ at 3 weeks. Wounds of all patients showed an obvious reduction in their size by 3-week treatment [127].

\section{Conclusion}

The nanofibers prepared from the renewable polymers can combine the excellent properties of the renewable polymers and nanofibers. They have become one of the most important materials used in wound dressing and attracted more and more attentions in recent years. The preparation methods, including electrospinning, bubbfil spinning, centrifugal spinning, and freeze-drying, have been widely used to prepare the nanofibers from the renewable polymers. Particularly the wound dressings prepared from chitosan, cellulose, alginate, gelatin, hyaluronic acid, collagen, and silk fibroin or their composites have been widely used as the wound dressing to promote wound healing, hemostasis, skin regeneration, diabetic ulcers, tissue engineering, and so on. Many of the dressings have been used in clinic. But for many renewable polymers which originated from natural components, there are many factors which influence the structure and property of the polymers and they are hard to be controlled. Although their properties are versatile, many of the properties are less prominent. When the renewable polymers are prepared to nanofibers and used for wound dressing, many of their properties, including antibacterial properties, hemostatic properties, biodegradability, cell adhesion and proliferation, and mechanical properties, need to be regulated by controlling the structure and properties of the nanofibers or compositing with other synthetic polymers to fit for the requirement of different wound. The above problems have become the future research keynotes for the nanofibers prepared from the renewable polymers.

\section{Competing Interests}

The authors declare that they have no competing interests.

\section{Acknowledgments}

This work was supported by the Youth Talent Plan of Beijing City, the Combination Project of Guangdong Province, and the "YangFan" Innovative Research Team Project of Guangdong Province.

\section{References}

[1] P. Scarfato, L. Di Maio, and L. Incarnato, "Recent advances and migration issues in biodegradable polymers from renewable sources for food packaging," Journal of Applied Polymer Science, vol. 132, no. 48, Article ID 42597, 2015.

[2] L. Yu, K. Dean, and L. Li, "Polymer blends and composites from renewable resources," Progress in Polymer Science, vol. 31, no. 6, pp. 576-602, 2006.

[3] J. J. Blaker, K.-Y. Lee, and A. Bismarck, "Hierarchical composites made entirely from renewable resources," Journal of Biobased Materials and Bioenergy, vol. 5, no. 1, pp. 1-16, 2011.

[4] V. Pillay, C. Dott, Y. E. Choonara et al., "A review of the effect of processing variables on the fabrication of electrospun nanofibers for drug delivery applications," Journal of Nanomaterials, vol. 2013, Article ID 789289, 22 pages, 2013.

[5] R. Nayak, R. Padhye, I. L. Kyratzis, Y. B. Truong, and L. Arnold, "Recent advances in nanofibre fabrication techniques," Textile Research Journal, vol. 82, no. 2, pp. 129-147, 2012.

[6] Y. J. Li, F. Chen, J. Nie, and D. Z. Yang, "Electrospun poly(lactic acid)/chitosan core-shell structure nanofibers from homogeneous solution," Carbohydrate Polymers, vol. 90, no. 4, pp. 14451451, 2012.

[7] O. Ayaz, N. Ucar, E. Bahar et al., "Properties of composite nanofiber produced by single and coaxial nozzle method used for electrospinning technique," International Journal of Chemical and Biological Engineering, vol. 6, pp. 52-56, 2012.

[8] S. Agarwal, A. Greiner, and J. H. Wendorff, "Functional materials by electrospinning of polymers," Progress in Polymer Science, vol. 38, no. 6, pp. 963-991, 2013.

[9] Z. J. Yu, Y. H. Lin, W. W. Huang et al., "Multi spinnerets electrospinning with assistant sheath gas," in Proceedings of the 9th IEEE International Conference on Nano/Micro Engineered and Molecular Systems, pp. 64-67, IEEE, Waikiki Beach, Hawaii, USA, April 2014.

[10] I. G. Kim, J.-H. Lee, A. R. Unnithan, C.-H. Park, and C. S. Kim, "A comprehensive electric field analysis of cylinder-type multi-nozzle electrospinning system for mass production of 
nanofibers," Journal of Industrial and Engineering Chemistry, vol. 31, pp. 251-256, 2015.

[11] A. Y. Nuryantini, M. M. Munir, M. P. Ekaputra, T. Suciati, and Khairurrijal, "Electrospinning of poly(vinyl alcohol)/chitosan via multi-nozzle spinneret and drum collector," Advanced Materials Research, vol. 896, pp. 41-44, 2014.

[12] N. Sasithorn and L. Martinová, "Fabrication of silk nanofibres with needle and roller electrospinning methods," Journal of Nanomaterials, vol. 2014, Article ID 947315, 9 pages, 2014.

[13] X. Wang, H. Niu, X. Wang, and T. Lin, "Needleless electrospinning of uniform nanofibers using spiral coil spinnerets," Journal of Nanomaterials, vol. 2012, Article ID 785920, 9 pages, 2012.

[14] R.-X. Chen, Y. Li, and J.-H. He, "Mini-review on Bubbfil spinning process for mass-production of nanofibers," Revista Materia, vol. 19, no. 4, Article ID 11560, pp. 325-344, 2014.

[15] J.-H. He, H.-Y. Kong, R.-R. Yang et al., "Review on fiber morphology obtained by bubble electrospinning and blown bubble spinning," Thermal Science, vol. 16, no. 5, pp. 1263-1279, 2012.

[16] Z.-B. Li, H.-Y. Liu, and H. Dou, "On air blowing direction in the blown bubble-spinning," Revista Materia, vol. 19, no. 4, pp. 345-349, 2014.

[17] J.-H. He and Y. Liu, "Control of bubble size and bubble number in bubble electrospinning," Computers and Mathematics with Applications, vol. 64, no. 5, pp. 1033-1035, 2012.

[18] G. J. Jiang, S. Zhang, Y. T. Wang, and X. H. Qin, "An improved free surface electrospinning with micro-bubble solution system for massive production of nanofibers," Materials Letters, vol. 144, pp. 22-25, 2015.

[19] H. Dou, B.-Q. Zuo, and J.-H. He, "Blown bubble-spinning for fabrication of superfine fibers," Thermal Science, vol. 16, no. 5, pp. 1465-1466, 2012.

[20] Y. Lu, Y. Li, S. Zhang et al., "Parameter study and characterization for polyacrylonitrile nanofibers fabricated via centrifugal spinning process," European Polymer Journal, vol. 49, no. 12, pp. 3834-3845, 2013.

[21] M. A. Hammami, M. Krifa, and O. Harzallah, "Centrifugal force spinning of PA6 nanofibers-processability and morphology of solution-spun fibers," The Journal of the Textile Institute, vol. 105, no. 6, pp. 637-647, 2014.

[22] A. E. Erickson, D. Edmondson, F.-C. Chang et al., "Highthroughput and high-yield fabrication of uniaxially-aligned chitosan-based nanofibers by centrifugal electrospinning," Carbohydrate Polymers, vol. 134, Article ID 10199, pp. 467-474, 2015.

[23] J. Wu and J. C. Meredith, "Assembly of chitin nanofibers into porous biomimetic structures via freeze drying," ACS Macro Letters, vol. 3, no. 2, pp. 185-190, 2014.

[24] N. Salamian, S. Irani, M. Zandi, S. M. Saeed, and S. M. Atyabi, "Cell attachment studies on electrospun nanofibrous PLGA and freeze-dried porous PLGA," Nano Bulletin, vol. 2, no. 1, Article ID 130103, 2013.

[25] G. Ma, Z. Wang, J. Chen, R. Yin, B. Chen, and J. Nie, "Freezedried chitosan-sodium hyaluronate polyelectrolyte complex fibers as tissue engineering scaffolds," New Journal of Chemistry, vol. 38, no. 3, pp. 1211-1217, 2014.

[26] M. Pakravan, M.-C. Heuzey, and A. Ajji, "A fundamental study of chitosan/PEO electrospinning," Polymer, vol. 52, no. 21, pp. 4813-4824, 2011.

[27] A. Sionkowska, "Current research on the blends of natural and synthetic polymers as new biomaterials: review," Progress in Polymer Science, vol. 36, no. 9, pp. 1254-1276, 2011.
[28] M. Dash, F. Chiellini, R. M. Ottenbrite, and E. Chiellini, "Chitosan-a versatile semi-synthetic polymer in biomedical applications," Progress in Polymer Science, vol. 36, no. 8, pp. 9811014, 2011.

[29] K. Ohkawa, K.-I. Minato, G. Kumagai, S. Hayashi, and H. Yamamoto, "Chitosan nanofiber," Biomacromolecules, vol. 7, no. 11, pp. 3291-3294, 2006.

[30] V. Sencadas, D. M. Correia, A. Areias et al., "Determination of the parameters affecting electrospun chitosan fiber size distribution and morphology," Carbohydrate Polymers, vol. 87, no. 2, pp. 1295-1301, 2012.

[31] M. Z. Elsabee, H. F. Naguib, and R. E. Morsi, "Chitosan based nanofibers, review," Materials Science and Engineering C, vol. 32, no. 7, pp. 1711-1726, 2012.

[32] P. Su, C. J. Wang, X. Y. Yang et al., "Electrospinning of chitosan nanofibers: the favorable effect of metal ions," Carbohydrate Polymers, vol. 84, no. 1, pp. 239-246, 2011.

[33] A. A. Nada, R. James, N. B. Shelke et al., "A smart methodology to fabricate electrospun chitosan nanofiber matrices for regenerative engineering applications," Polymers for Advanced Technologies, vol. 25, no. 5, pp. 507-515, 2014.

[34] R. Zhao, X. Li, B. Sun et al., "Electrospun chitosan/sericin composite nanofibers with antibacterial property as potential wound dressings," International Journal of Biological Macromolecules, vol. 68, pp. 92-97, 2014.

[35] Y. H. Zhao, Y. Zhou, X. M. Wu, L. Wang, L. Xu, and S. C. Wei, "A facile method for electrospinning of Ag nanoparticles/poly (vinyl alcohol)/carboxymethyl-chitosan nanofibers," Applied Surface Science, vol. 258, no. 22, pp. 8867-8873, 2012.

[36] R. Liu, X. Xu, X. Zhuang, and B. Cheng, "Solution blowing of chitosan/PVA hydrogel nanofiber mats," Carbohydrate Polymers, vol. 101, no. 1, pp. 1116-1121, 2014.

[37] A. M. Abdelgawad, S. M. Hudson, and O. J. Rojas, "Antimicrobial wound dressing nanofiber mats from multicomponent (chitosan/silver-NPs/polyvinyl alcohol) systems," Carbohydrate Polymers, vol. 100, pp. 166-178, 2014.

[38] H. Y. Ma, C. Burger, B. S. Hsiao, and B. Chu, "Fabrication and characterization of cellulose nanofiber based thin-film nanofibrous composite membranes," Journal of Membrane Science, vol. 454, pp. 272-282, 2014.

[39] L. Fu, J. Zhang, and G. Yang, "Present status and applications of bacterial cellulose-based materials for skin tissue repair," Carbohydrate Polymers, vol. 92, no. 2, pp. 1432-1442, 2013.

[40] L. M. M. Costa, G. M. Olyveira, P. Basmaji, and L. X. Filho, "Nanopores structure in electrospun bacterial cellulose," Journal of Biomaterials and Nanobiotechnology, vol. 3, pp. 92-96, 2012.

[41] N. Shah, M. Ul-Islam, W. A. Khattak, and J. K. Park, "Overview of bacterial cellulose composites: a multipurpose advanced material," Carbohydrate Polymers, vol. 98, no. 2, pp. 1585-1598, 2013.

[42] P. Chen, Y. S. Yun, H. Bak, S. Y. Cho, and H.-J. Jin, "Multiwalled carbon nanotubes-embedded electrospun bacterial cellulose nanofibers," Molecular Crystals and Liquid Crystals, vol. 519, pp. 169-178, 2010.

[43] E. Omollo, C. Zhang, J. I. Mwasiagi, and S. Ncube, "Electrospinning cellulose acetate nanofibers and a study of their possible use in high-efficiency filtration," Journal of Industrial Textiles, 2014.

[44] A. Khalf, K. Singarapu, and S. V. Madihally, "Cellulose acetate core-shell structured electrospun fiber: fabrication and characterization," Cellulose, vol. 22, no. 2, pp. 1389-1400, 2015. 
[45] K. T. Shalumon, K. H. Anulekha, S. V. Nair, S. V. Nair, K. P. Chennazhi, and R. Jayakumar, "Sodium alginate/poly(vinyl alcohol)/nano $\mathrm{ZnO}$ composite nanofibers for antibacterial wound dressings," International Journal of Biological Macromolecules, vol. 49, no. 3, pp. 247-254, 2011.

[46] N. Bhattarai and M. Zhang, "Controlled synthesis and structural stability of alginate-based nanofibers," Nanotechnology, vol. 18, no. 45, Article ID 455601, 2007.

[47] S. A. Stone, P. Gosavi, T. J. Athauda, and R. R. Ozer, "In situ citric acid crosslinking of alginate/polyvinyl alcohol electrospun nanofibers," Materials Letters, vol. 112, pp. 32-35, 2013.

[48] V. Leung, R. Hartwell, S. S. Elizei, H. J. Yang, A. Ghahary, and F. Ko, "Postelectrospinning modifications for alginate nanofiber-based wound dressings," Journal of Biomedical Materials Research Part B Applied Biomaterials, vol. 102, no. 3, pp. 508-515, 2014.

[49] K. Y. Lee, L. Jeong, Y. O. Kang, S. J. Lee, and W. H. Park, "Electrospinning of polysaccharides for regenerative medicine," Advanced Drug Delivery Reviews, vol. 61, no. 12, pp. 1020-1032, 2009.

[50] D. W. Fang, Y. Liu, S. Jiang, J. Nie, and G. P. Ma, "Effect of intermolecular interaction on electrospinning of sodium alginate," Carbohydrate Polymers, vol. 85, no. 1, pp. 276-279, 2011.

[51] G. P. Ma, D. W. Fang, Y. Liu, X. D. Zhu, and J. Nie, "Electrospun sodium alginate/poly(ethylene oxide) core-shell nanofibers scaffolds potential for tissue engineering applications," Carbohydrate Polymers, vol. 87, no. 1, pp. 737-743, 2012.

[52] S. A. Park, K. E. Park, and W. D. Kim, "Preparation of sodium alginate/poly(ethylene oxide) blend nanofibers with lecithin," Macromolecular Research, vol. 18, no. 9, pp. 891-896, 2010.

[53] S. Safi, M. Morshed, S. A. H. Ravandi, and M. Ghiaci, "Study of electrospinning of sodium alginate, blended solutions of sodium alginate/poly(vinyl alcohol) and sodium alginate/poly(ethylene oxide)," Journal of Applied Polymer Science, vol. 104, no. 5, pp. 3245-3255, 2007.

[54] V. M. Merkle, P. L. Tran, M. Hutchinson et al., "Core-shell PVA/gelatin electrospun nanofibers promote human umbilical vein endothelial cell and smooth muscle cell proliferation and migration," Acta Biomaterialia, vol. 27, pp. 77-87, 2015.

[55] R.-Y. Tsai, S.-C. Hung, J.-Y. Lai, D.-M. Wang, and H.-J. Hsieh, "Electrospun chitosan-gelatin-polyvinyl alcohol hybrid nanofibrous mats: production and characterization," Journal of the Taiwan Institute of Chemical Engineers, vol. 45, no. 4, pp. 19751981, 2014.

[56] M. Fallah, S. H. Bahrami, and R. M. Marziyeh, "Fabrication and characterization of PCL/gelatin/curcumin nanofibers and their antibacterial properties," Journal of Industrial Textiles, pp. 1-16, 2015.

[57] K. Jalaja, P. R. A. Kumar, T. Dey, S. C. Kundu, and N. R. James, "Modified dextran cross-linked electrospun gelatin nanofibres for biomedical applications," Carbohydrate Polymers, vol. 114, pp. 467-475, 2014.

[58] T. H. Nguyen and B. T. Lee, "Fabrication and characterization of cross-linked gelatin electro-spun nano-fibers," Journal of Biomedical Science and Engineering, vol. 3, no. 12, pp. 1117-1124, 2010.

[59] Y. Z. Zhang, J. Venugopal, Z.-M. Huang, C. T. Lim, and S. Ramakrishna, "Crosslinking of the electrospun gelatin nanofibers,” Polymer, vol. 47, no. 8, pp. 2911-2917, 2006.
[60] J. H. Ko, H. Yin, J. An et al., “Characterization of cross-linked gelatin nanofibers through electrospinning," Macromolecular Research, vol. 18, no. 2, pp. 137-143, 2010.

[61] Z.-M. Huang, Y. Z. Zhang, S. Ramakrishna, and C. T. Lim, "Electrospinning and mechanical characterization of gelatin nanofibers," Polymer, vol. 45, no. 15, pp. 5361-5368, 2004.

[62] C. S. Ki, D. H. Baek, K. D. Gang, K. H. Lee, I. C. Um, and Y. H. Park, "Characterization of gelatin nanofiber prepared from gelatin-formic acid solution," Polymer, vol. 46, no. 14, pp. 5094$5102,2005$.

[63] J.-H. Song, H.-E. Kim, and H.-W. Kim, "Production of electrospun gelatin nanofiber by water-based co-solvent approach," Journal of Materials Science: Materials in Medicine, vol. 19, no. 1, pp. 95-102, 2008.

[64] K. Sisson, C. Zhang, M. C. Farach-Carson, D. B. Chase, and J. F. Rabolt, "Evaluation of cross-linking methods for electrospun gelatin on cell growth and viability," Biomacromolecules, vol. 10, no. 7, pp. 1675-1680, 2009.

[65] W. P. Lu, M. Ma, H. T. Xu, B. Zhang, X. F. Cao, and Y. C. Guo, "Gelatin nanofibers prepared by spiral-electrospinning and cross-linked by vapor and liquid-phase glutaraldehyde," Materials Letters, vol. 140, pp. 1-4, 2015.

[66] P. Slemming-Adamsen, J. Song, M. Dong, F. Besenbacher, and M. Chen, "In situ cross-linked PNIPAM/gelatin nanofibers for thermo-responsive drug release," Macromolecular Materials and Engineering, vol. 300, no. 12, pp. 1226-1231, 2015.

[67] H. W. Kwak, M. J. Kang, J. H. Bae et al., "Fabrication of Phaeodactylum tricornutum extract-loaded gelatin nanofibrous mats exhibiting antimicrobial activity," International Journal of Biological Macromolecules, vol. 63, pp. 198-204, 2014.

[68] Y.-H. Shan, L.-H. Peng, X. Liu, X. Chen, J. Xiong, and J.Q. Gao, "Silk fibroin/gelatin electrospun nanofibrous dressing functionalized with astragaloside IV induces healing and antiscar effects on burn wound," International Journal of Pharmaceutics, vol. 479, no. 2, pp. 291-301, 2015.

[69] X. T. Han, Z. G. Xing, S. X. Si, Y. Y. Yao, and Q. Y. Zhang, "Electrospun grape seed polyphenols/gelatin composite fibers contained silver nanoparticles as biomaterials," Fibers and Polymers, vol. 15, no. 12, pp. 2572-2580, 2014.

[70] E. K. Brenner, J. D. Schiffman, E. A. Thompson, L. J. Toth, and C. L. Schauer, "Electrospinning of hyaluronic acid nanofibers from aqueous ammonium solutions," Carbohydrate Polymers, vol. 87, no. 1, pp. 926-929, 2012.

[71] C.-H. Chen, S.-H. Chen, K. T. Shalumon, and J.-P. Chen, "Dual functional core-sheath electrospun hyaluronic acid/ polycaprolactone nanofibrous membranes embedded with silver nanoparticles for prevention of peritendinous adhesion," Acta Biomaterialia, vol. 26, pp. 225-235, 2015.

[72] R. Uppal, G. N. Ramaswamy, C. Arnold, R. Goodband, and Y. Wang, "Hyaluronic acid nanofiber wound dressingproduction, characterization, and in vivo behavior," Journal of Biomedical Materials Research Part B: Applied Biomaterials, vol. 97, no. 1, pp. 20-29, 2011.

[73] Z. G. Chen, P. W. Wang, B. Wei, X. M. Mo, and F. Z. Cui, "Electrospun collagen-chitosan nanofiber: a biomimetic extracellular matrix for endothelial cell and smooth muscle cell," Acta Biomaterialia, vol. 6, no. 2, pp. 372-382, 2010.

[74] K. S. Rho, L. Jeong, G. Lee et al., "Electrospinning of collagen nanofibers: effects on the behavior of normal human keratinocytes and early-stage wound healing," Biomaterials, vol. 27, no. 8, pp. 1452-1461, 2006. 
[75] S.-J. Liu, Y.-C. Kau, C.-Y. Chou, J.-K. Chen, R.-C. Wu, and W.L. Yeh, "Electrospun PLGA/collagen nanofibrous membrane as early-stage wound dressing," Journal of Membrane Science, vol. 355, no. 1-2, pp. 53-59, 2010.

[76] L. Huang, K. Nagapudi, P. R. Apkarian, and E. L. Chaikof, "Engineered collagen-PEO nanofibers and fabrics," Journal of Biomaterials Science-Polymer Edition, vol. 12, no. 9, pp. 979993, 2001.

[77] E. Schnell, K. Klinkhammer, S. Balzer et al., "Guidance of glial cell migration and axonal growth on electrospun nanofibers of poly- $\varepsilon$-caprolactone and a collagen/poly- $\varepsilon$-caprolactone blend," Biomaterials, vol. 28, no. 19, pp. 3012-3025, 2007.

[78] J. S. Choi, S. J. Lee, G. J. Christ, A. Atala, and J. J. Yoo, “The influence of electrospun aligned poly( $\varepsilon$-caprolactone)/collagen nanofiber meshes on the formation of self-aligned skeletal muscle myotubes," Biomaterials, vol. 29, no. 19, pp. 2899-2906, 2008.

[79] I. K. Kwon and T. Matsuda, "Co-electrospun nanofiber fabrics of poly (L-lactide-co- $\epsilon$-caprolactone) with type I collagen or heparin," Biomacromolecules, vol. 6, no. 4, pp. 2096-2105, 2005.

[80] J. Venugopal, S. Low, A. T. Choon, T. S. S. Kumar, and S. Ramakrishna, "Mineralization of osteoblasts with electrospun collagen/hydroxyapatite nanofibers," Journal of Materials Science: Materials in Medicine, vol. 19, no. 5, pp. 2039-2046, 2008.

[81] J.-H. Song, H.-E. Kim, and H.-W. Kim, "Electrospun fibrous web of collagen-apatite precipitated nanocomposite for bone regeneration," Journal of Materials Science: Materials in Medicine, vol. 19, no. 8, pp. 2925-2932, 2008.

[82] W. He, T. Yong, W. E. Teo, Z. Ma, and S. Ramakrishna, "Fabrication and endothelialization of collagen-blended biodegradable polymer nanofibers: potential vascular graft for blood vessel tissue engineering," Tissue Engineering, vol. 11, no. 9-10, pp. 1574-1588, 2005.

[83] J.-P. Chen, G.-Y. Chang, and J.-K. Chen, "Electrospun collagen/chitosan nanofibrous membrane as wound dressing," Colloids and Surfaces A: Physicochemical and Engineering Aspects, vol. 313-314, pp. 183-188, 2008.

[84] T. Liu, J. Xu, B. P. Chan, and S. Y. Chew, "Sustained release of neurotrophin-3 and chondroitinase $\mathrm{ABC}$ from electrospun collagen nanofiber scaffold for spinal cord injury repair," Journal of Biomedical Materials Research Part A, vol. 100, no. 1, pp. 236242, 2011.

[85] S. H. Kim, Y. S. Nam, T. S. Lee, and W. H. Park, "Silk fibroin nanofiber. Electrospinning, properties, and structure," Polymer Journal, vol. 35, no. 2, pp. 185-190, 2003.

[86] L. Jeong, K. Y. Lee, J. W. Liu, and W. H. Park, “Time-resolved structural investigation of regenerated silk fibroin nanofibers treated with solvent vapor," International Journal of Biological Macromolecules, vol. 38, no. 2, pp. 140-144, 2006.

[87] B.-M. Min, L. Jeong, K. Y. Lee, and W. H. Park, "Regenerated silk fibroin nanofibers: water vapor-induced structural changes and their effects on the behavior of normal human cells," Macromolecular Bioscience, vol. 6, no. 4, pp. 285-292, 2006.

[88] L. Jeong, D. Cho, O. H. Kwon, B.-M. Min, and W. H. Park, "Cellular response of silk fibroin nanofibers containing silver nanoparticles in vitro," Macromolecular Research, vol. 22, no. 7, pp. 796-803, 2014.

[89] S. Shahverdi, M. Hajimiri, M. A. Esfandiari et al., "Fabrication and structure analysis of poly(lactide-co-glycolic acid)/silk fibroin hybrid scaffold for wound dressing applications," International Journal of Pharmaceutics, vol. 473, no. 1-2, pp. 345-355, 2014.
[90] S. Çalamak, C. Erdoğdu, M. Özalp, and K. Ulubayram, "Silk fibroin based antibacterial bionanotextiles as wound dressing materials," Materials Science and Engineering C, vol. 43, pp. 1120, 2014

[91] J. Chutipakdeevong, U. Ruktanonchai, and P. Supaphol, "Hybrid biomimetic electrospun fibrous mats derived from $\operatorname{poly}(\varepsilon-$ caprolactone) and silk fibroin protein for wound dressing application," Journal of Applied Polymer Science, vol. 132, no. 11, Article ID 41653, 2015.

[92] S. Calamak, E. A. Aksoy, C. Erdogdu, M. Sagıroglu, and K. Ulubayram, "Silver nanoparticle containing silk fibroin bionanotextiles," Journal of Nanoparticle Research, vol. 17, no. 2, pp. 1-9, 2015.

[93] K. Wei, Y. Li, K.-O. Kim et al., "Fabrication of nanohydroxyapatite on electrospun silk fibroin nanofiber and their effects in osteoblastic behavior," Journal of Biomedical Materials Research Part A, vol. 97, no. 3, pp. 272-280, 2011.

[94] C. S. Ki, E. H. Gang, I. C. Um, and Y. H. Park, "Nanofibrous membrane of wool keratose/silk fibroin blend for heavy metal ion adsorption," Journal of Membrane Science, vol. 302, no. 1-2, pp. 20-26, 2007.

[95] C. S. Jiang and X. B. Chen, "Research process in the preparation of biomedical dressings by electrospinning," Synthetic Fiber Industry, vol. 35, no. 3, pp. 44-46, 2012.

[96] T. T. T. Nguyen, C. Ghosh, S.-G. Hwang, L. D. Tran, and J. S. Park, "Characteristics of curcumin-loaded poly (lactic acid) nanofibers for wound healing," Journal of Materials Science, vol. 48, no. 20, pp. 7125-7133, 2013.

[97] Y.-F. Goh, I. Shakir, and R. Hussain, "Electrospun fibers for tissue engineering, drug delivery, and wound dressing," Journal of Materials Science, vol. 48, no. 8, pp. 3027-3054, 2013.

[98] T. Wu, "Absorbable composite dressing," China, CN102008740A, CN102008740-B.

[99] J. Fang, H. Niu, T. Lin, and X. Wang, "Applications of electrospun nanofibers," Chinese Science Bulletin, vol. 53, no. 15, pp. 2265-2286, 2008.

[100] X.-F. Huang, J.-W. Jia, Z.-K. Wang, and Q.-L. Hu, "A novel chitosan-based sponge coated with self-assembled thrombin/tannic acid multilayer films as a hemostatic dressing," Chinese Journal of Polymer Science, vol. 33, no. 2, pp. 284-290, 2015.

[101] C. Theisen, S. F. Winkelmann, K. Knappstein et al., "Influence of nanofibers on growth and gene expression of human tendon derived fibroblast," BioMedical Engineering Online, vol. 9, article 9, 2010.

[102] M. Dubský, Š. Kubinová, J. Širc et al., "Nanofibers prepared by needleless electrospinning technology as scaffolds for wound healing," Journal of Materials Science: Materials in Medicine, vol. 23, no. 4, pp. 931-941, 2012.

[103] M. A. de Moraes and M. M. Beppu, "Biocomposite membranes of sodium alginate and silk fibroin fibers for biomedical applications," Journal of Applied Polymer Science, vol. 130, no. 5, pp. 3451-3457, 2013.

[104] Z. X. Zhou, X. L. Wei, J. R. Yao et al., "Evaluation of biological hemostatic dressings and gauzes in surgical wounds," Journal of Clinical Rehabilitative Tissue Engineering Research, vol. 14, no. 51, pp. 9635-9638, 2010.

[105] P. Jiang, "Preparation of the PLLA from the fibrinogen and applications in the wound bleeding pig model," Journal of Trauma and Emergency, vol. 1, no. 4, p. 54, 2013. 
[106] X. Zhao, X. H. Cao, Y. Y. Ma et al., "Hemostatic effect of fibrin hemostatic dressing," China Blood Transfusion, vol. 23, no. 4, pp. 250-252, 2010.

[107] K. Y. Baik, S. Y. Park, S. Namgung et al., "Synthetic nanowire/nanotube-based solid substrates for controlled cell growth," Nano Convergence, vol. 1, article 28, 2014.

[108] T. Rajangam, H.-J. Paik, and S. S. A. An, "Development of fibrinogen microspheres as a biodegradable carrier for tissue engineering," Biochip Journal, vol. 5, no. 2, pp. 175-183, 2011.

[109] R. G. Pillai and Z. A. Naieb, "Plastibell circumcision supported by a calcium-alginate fibre dressing to reduce bleeding," Arab Journal of Urology, vol. 13, no. 3, pp. 179-181, 2015.

[110] B. Sibaja, E. Culbertson, P. Marshall et al., "Preparation of alginate-chitosan fibers with potential biomedical applications," Carbohydrate Polymers, vol. 134, pp. 598-608, 2015.

[111] W. S. Liu, B. Q. Han, and Q. S. Gu, "Preparation method and application of the hemostasis and healing materials from the water soluble chitosan fibres," CN 200710015743.6, 2007.

[112] H. M. Wang, J. Y. Pan, B. Yang, and K. X. Liu, "Animal test and clinical application of total soluble hemostatic fiber," Journal of Clinical Pharmacology and Therapeutics in China, vol. 11, no. 9, pp. s1017-s1020, 2006.

[113] J. Z. Niu, B. P. Li, H. T. Lan, H. Wang, and K. Li, "Effect of lidocaine gelatin fiber net on the hemostatic and analgesic effect of endoscopic sinus surgery," Chinese Journal of Primary Medicine and Pharmacy, vol. 20, no. 12, pp. 1776-1777, 2013.

[114] S. Liu, J. Z. Niu, H. J. Ge et al., "Clinical observation of the hemostatic effect of gelatin fiber net," Journal of Chinese Trauma, vol. 16, no. 3, p. 181, 2000.

[115] D. A. Das, K. A. Grimmer, A. L. Sparnon, S. E. McRae, and B. H. Thomas, "The efficacy of playing a virtual game in modulating pain for children with a cuteburn injuries: a randomized controlled trial," BMC Pediatrics, vol. 5, article 1, 2005.

[116] S. C. Sharma, M. M. Bagree, A. L. Bhat, B. B. Banga, and M. P. Singh, "Amniotic membrane is an effective burn dressing material," The Japanese Journal of Surgery, vol. 15, no. 2, pp. 140$143,1985$.

[117] G. D. Mogoşanu and A. M. Grumezescu, "Natural and synthetic polymers for wounds and burns dressing," International Journal of Pharmaceutics, vol. 463, no. 2, pp. 127-136, 2014.

[118] E. G. Kee, R. M. Kimble, L. Cuttle, and K. Stockton, "Comparison of three different dressings for partial thickness burns in children: study protocol for a randomised controlled trial," Trials, vol. 14, article 403, 8 pages, 2013.

[119] J. Venugopal and S. Ramakrishna, "Applications of polymer nanofibers in biomedicine and biotechnology," Applied Biochemistry and Biotechnology, vol. 125, no. 3, pp. 147-157, 2005.

[120] L. Y. Kossovich, Y. Salkovskiy, and I. V. Kirillova, "Electrospun chitosan nanofiber materials as burn dressing," in Proceedings of the 6th World Congress of Biomechanics (WCB '10), C. T. Lim and J. C. H. Goh, Eds., vol. 31 of IFMBE Proceedings, pp. 12121214, Singapore, August 2010.

[121] O. Chaisiri, N. Chanunpanich, and B. S. Hanpanich, "Polycarpolactone fibers gellation with gelatin ground substance: Engineered skin extracellular matrix aims for using as tissue engineering skin," in The 15th International Conference on Biomedical Engineering: ICBME 2013, 4th to 7th December 2013, Singapore, vol. 43 of IFMBE Proceedings, pp. 267-270, Springer, Berlin, Germany, 2014.
[122] W. Hu, Z.-M. Huang, S.-Y. Meng, and C.-L. He, "Fabrication and characterization of chitosan coated braided PLLA wire using aligned electrospun fibers," Journal of Materials Science: Materials in Medicine, vol. 20, no. 11, pp. 2275-2284, 2009.

[123] N. Alhusein, I. S. Blagbrough, M. L. Beeton, A. Bolhuis, and P. A. D. Bank, "Electrospun zein/PCL fibrous matrices release tetracycline in a controlled manner, killing Staphylococcus aureus both in biofilms and ex vivo on pig skin, and are compatible with human skin cells," Pharmaceutical Research, vol. 33, no. 1, pp. 237-246, 2016.

[124] J.-B. Li, J. Han, and J. Ren, "Interaction of human fibroblasts with electrospun composites gelatin/PLLA, chitosan/PLLA and PLLA fibrous scaffolds," Journal of Shanghai Jiaotong University (Science), vol. 17, no. 5, pp. 559-566, 2012.

[125] J. C. Dumville, M. O. Soares, S. O'meara, and N. Cullum, "Systematic review and mixed treatment comparison: dressings to heal diabetic Foot ulcers," Diabetologia, vol. 55, no. 7, pp. 1902-1910, 2012.

[126] X. Wang, D. Zhang, and F. Shi, "Wound dressing useful for treating venous stasis ulcers and etc," China, WO2012092812-A1 CN102580135-A.

[127] J. L. Lázaro-Martínez, A. Cecilia-Matilla, J. Aragón-Sánchez, E. García-Morales, Y. Garcia-Alvarez, and F. Alvaro-Afonso, "Treatment of infected diabetic foot ulcers clinical effectiveness of a dressing of alginate and hydrocolloid, with silver fiber. Analysis of results of a series of cases," Revista de Enfermería, vol. 36, no. 11, pp. 29-34, 2013. 

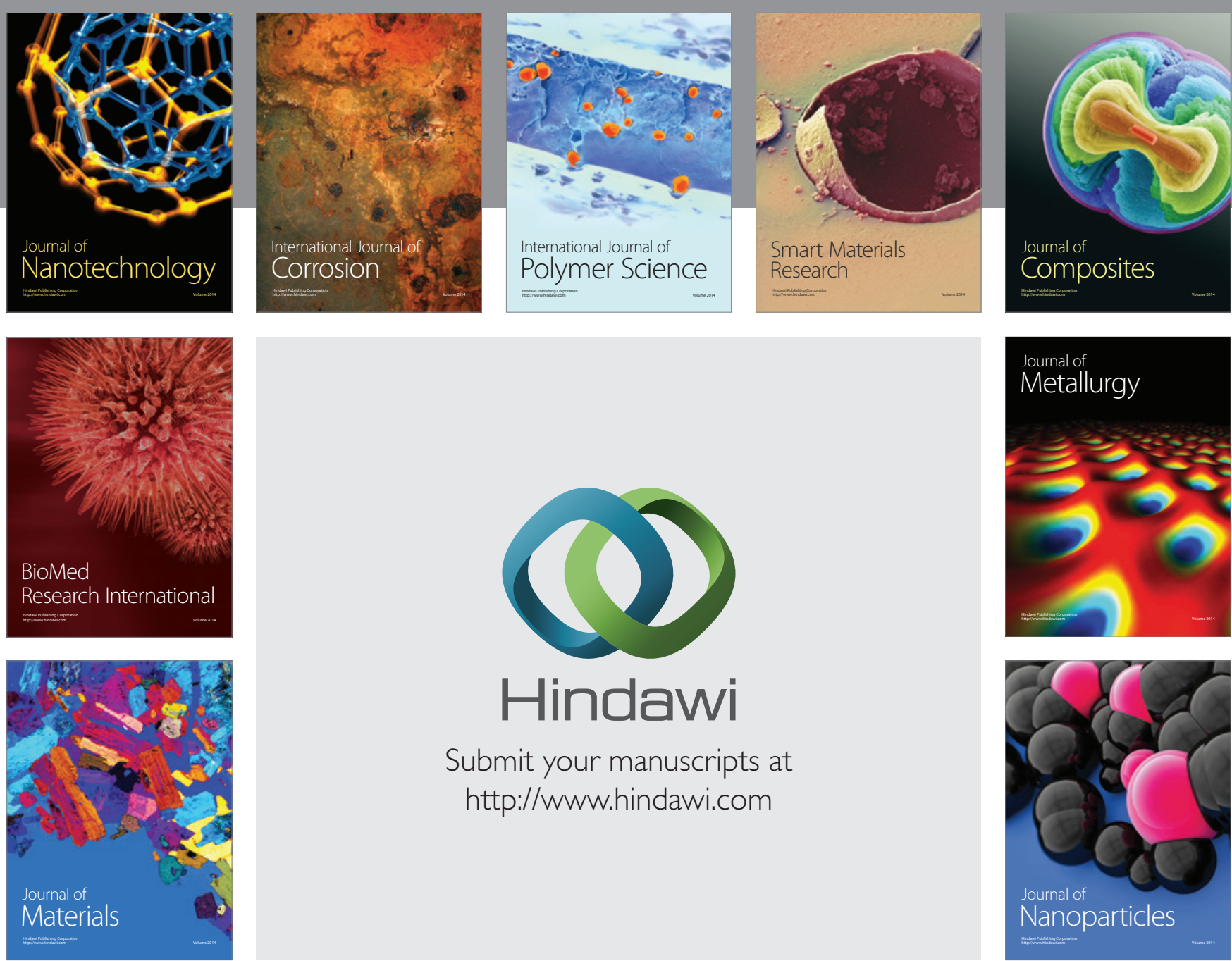

\section{Hindawi}

Submit your manuscripts at

http://www.hindawi.com

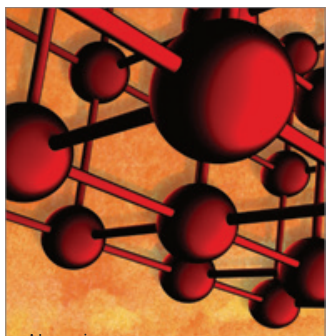

Materials Science and Engineering
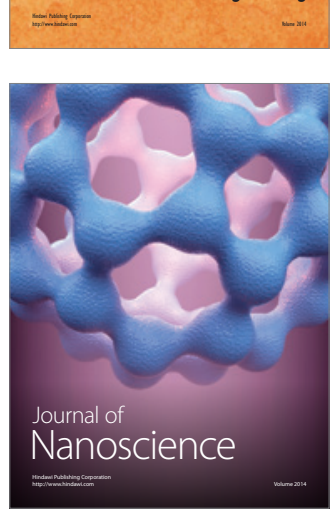
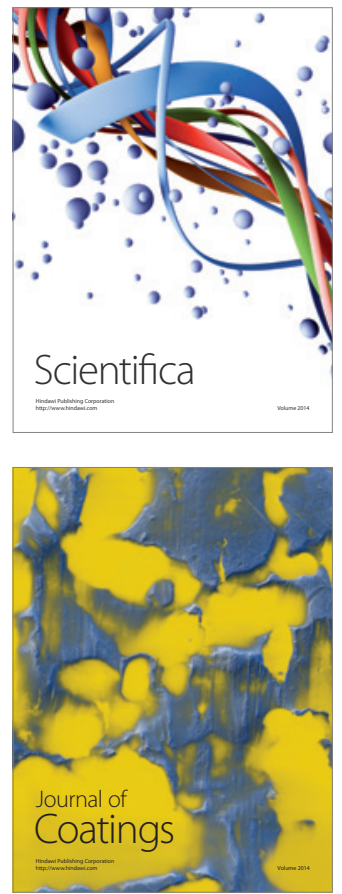
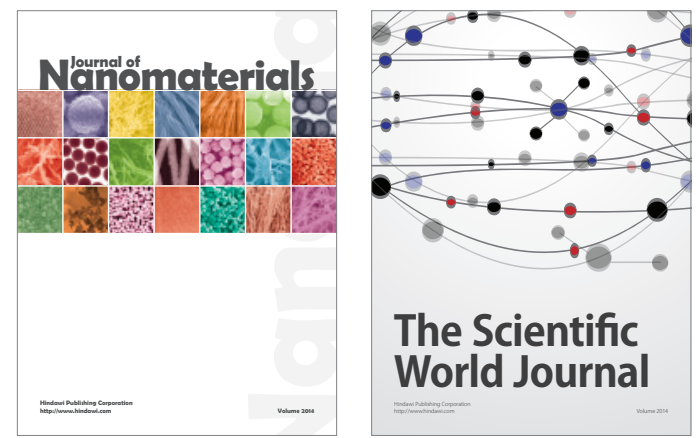

The Scientific World Journal
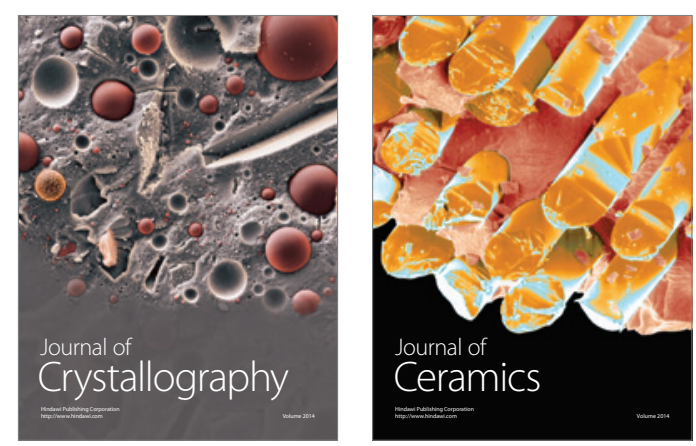
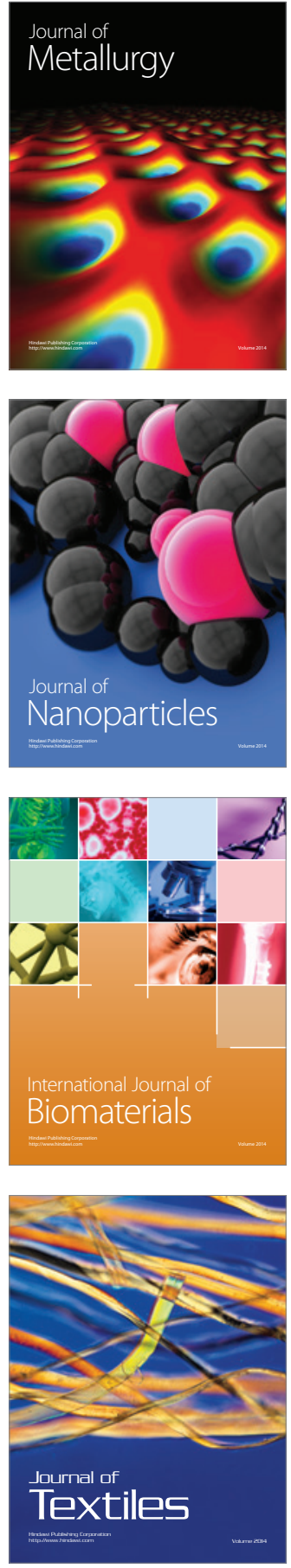